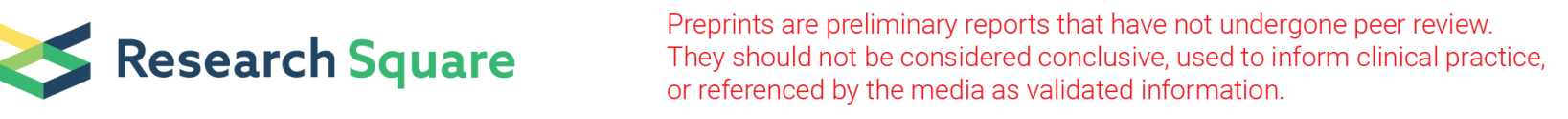

\title{
Construction of IncRNA-miRNA-mRNA regulatory network and correlation between prognostic biomarker PDGFRB and immune infiltrates in stomach adenocarcinoma
}

\author{
Guang Yang ( $\nabla$ 284554605@qq.com ) \\ Okayama University \\ Ying Wu \\ Mudanjiang Medical University \\ yi Ru Zheng \\ The mine hospital of xuzhou \\ shun Zai Jin \\ Mudanjiang Medical University \\ Bin Yan \\ Mudanjiang Medical University
}

\section{Research Article}

Keywords: PDGFRB, stomach adenocarcinoma, competing endogenous RNA network, long noncoding RNA, survival analysis

Posted Date: February 28th, 2022

DOl: https://doi.org/10.21203/rs.3.rs-1338495/v1

License: (c) (7) This work is licensed under a Creative Commons Attribution 4.0 International License. Read Full License 


\section{Abstract}

Purpose: Gastric cancer is a highly malignant and heterogeneous tumour with a poor prognosis and a high recurrence rate. Overexpressed PDGFRB has a correlation with poor survival outcomes in several malignancies. The objective of our study was to evaluate the prognostic value of PDGFRB and identify a IncRNA-miRNA-mRNA ceRNA network involved in stomach adenocarcinoma.

Methods: RNA-sequencing data, miRNA-sequencing data and corresponding patient clinical information were collected from the TCGA database. All statistical analysis was performed through the edgeR package. ENCORI database was applied to screen out miRNAs and IncRNAs interacting with the target gene. A IncRNA-miRNA-mRNA ceRNA network was constructed through Cytoscape. For DEmiRNAs, DElncRNAs and mRNAðthe differences between the overall survival of high-expression and low-expression group were evaluated by R language. TIMER 2.0 and GEPIA database were used to evaluate the value of $P D G F R B$ in immune cell infiltration and immunotherapy.

Results: Compared with normal tissue samples, PDGFRB was significantly upregulated in stomach adenocarcinoma. The upregulated expression of PDGFRB was significantly related to histologic grade, topography and clinical stage. LncRNA CASC15 and has-miR-30e-5p were identified to have correlations with PDGFRB. Patients with an elevated PDGFRB, IncRNA CASC15 and a decreased has-miR-30e-5p had a poor prognosis, in which elevated PDGFRB was an independent poor prognostic factor. In addition, the positive associations among PDGFRB, tumor infiltrating immune cells and immunotherapy were also observed.

Conclusions: An increased PDGFRB may serve as a promising and potential independent prognostic biomarker of a poor survival and therapeutic targets in stomach adenocarcinoma.

\section{Introduction}

Gastric cancer is a malignant tumour with aggressiveness as well as a high degree of malignancy and heterogeneity. Gastric cancer is the leading cause of cancer death, ranking fifth in cancer incidence and third in cancer-related mortality worldwide (Wang et al. 2020). However, the distribution of the incidence of gastric cancer is uneven. The incidence in Asia is much higher than that in Western countries. Eastern Asian countries account for approximately half of newly diagnosed gastric cancer cases worldwide. The occurrence of gastric cancer is closely related to many factors, such as genetic factors, environmental factors, dietary habits (nitrites and salted foods) and Helicobacter pylori infection (Johnston and Beckman 2019; Machlowska et al. 2020). Due to improved food preservation technology and the eradication of Helicobacter pylori, the morbidity of gastric cancer has declined. Gastric cancer is divided into three subtypes: adenocarcinoma, undifferentiated carcinoma and signet ring cell carcinoma (Sitarz et al. 2018). Stomach adenocarcinoma (STAD) represents the vast majority of gastric malignancies. Surgical treatment is the only measure for early gastric cancer. However, the treatment of advanced gastric cancer is still challenging, and the prognosis is not ideal (Petryszyn et al. 2020). Patients with stage $\nabla$ disease undergoing surgical treatment have a dismal 5-year survival rate between $18 \%$ and $50 \%$ according to the database (Sexton et al. 2020). Therefore, it is urgent to seek more effective molecular-driven therapeutic strategies and effective prognostic markers.

The platelet-derived growth factor receptor beta $(P D G F R B)$ gene is located on chromosome 5q31-q32, through which the receptors for platelet-derived growth factor $B$ (PDGFB) and platelet-derived growth factor D (PDGFD) are encoded (Dachy et al. 2019; Kim et al. 2008). PDGFRB is highly expressed in neurons, vascular smooth muscle cells, fibroblasts and pericytes (Mathorne et al. 2019). Frequent PDGFRB gene phenotype variants have been identified in several diseases (Hassan et al. 2019; Karasozen et al. 2019; Li et al. 2019; Mathorne et al. 2019). Among patients with head and neck squamous cell carcinoma, overexpressed PDGFRB had a correlation with poor survival outcomes (Lecerf et al. 2019). Recently, Ning Zhang et al. reported that the expression level of PDGFRB in tumor tissues was associated with TNM stage and lymph node 
metastasis of Chinese patients with non-small cell lung cancer (NSCLC) patients, which might exert a pivotal role in the progression of NSCLC (Zhang et al. 2018).

In 2011, Salmena et al. proposed a hypothesis on competing endogenous RNAs (ceRNAs) (Salmena et al. 2011), which postulated that RNA molecules could communicate with other RNAs through shared microRNA (miRNA) response elements. message RNAs (mRNAs), long noncoding RNAs (IncRNAs) and RNA transcripts can act as endogenous miRNA sponges to inhibit functions of miRNAs, thus affecting their expression together with that of downstream target genes (Fan and Liu 2018). A IncRNA is a kind of noncoding RNA, with a length of about 200 nt and no protein coding function, which has attracted increasing research interest recently (Mendell 2016; Quinn and Chang 2016). These RNAs participate in important biological processes by regulating gene expression at the transcriptional, post transcriptional and epigenetic level (Kornienko et al. 2013; Mercer and Mattick 2013; Shi et al. 2013). Recent studies have provided emerging support for the involvement of the ceRNA activity of IncRNAs in the progression of various types of cancers. For example, in oral cancer, IncRNA HCG22 has a potential as a diagnostic and prognostic marker, and the low expression of IncRNA HCG22 is significantly associated with a reduced patient survival rate (Yin et al. 2020). Zhengwei Chen et al. found that IncRNA DSCR8 could adsorb miR-137 to reduce its inhibitory effect on the expression of CDC42, so as to promote the progression of gastric cancer cells and regulate cell cycle (Chen et al. 2021). However, in gastric cancer, IncRNA-miRNA-PDGFRB axis, which regulates the expression of PDGFRB, has not been reported.

Therefore, the purpose of our current research was to explore the correlation between PDGFRB and the clinicopathological characteristics of STAD, the role and potential prognostic value of PDGFRB based on The Cancer Genome Atlas (TCGA) database and construct IncRNA-miRNA-mRNA regulatory network. The research is expected to help to further elucidate the detailed molecular mechanism involved in tumorigenesis and progression of gastric cancer. Finally, we also examined the correlation between PDGFRB and tumour-infiltrating immune cells using TIMER.

\section{Results}

\section{Clinicopathological characteristics of STAD patients}

The clinical characteristics of the STAD patients, including age, sex, histological grade, clinical stage, primary tumour T, lymph node $\mathrm{N}$, metastasis $\mathrm{M}$, and survival status, were downloaded from the TCGA database (Table 1). The median age at diagnosis is 67 years. A total of 134 female and 241 male patients were involved in the current study. Tumours were divided into three grades. G1, G2 and G3 to include 10 (2.73\%), 137 (37.43\%), and 219 (59.84\%) cases, respectively. A total of 352

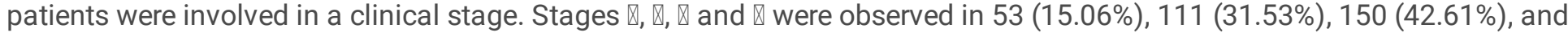
38 (10.80\%) patients, respectively. Topography contained 26.98\% $(n=99)$ T1-T2 and 73.02\% $(n=268)$ T3-T4. Of 357 patients, $246(68.91 \%)$ had lymph node metastasis, and 25 of 355 patients (7.04\%) had distant metastasis. The survival status of most patients was alive, accounting for $65.07 \%(n=244)$. 
Table 1

Clinical characteristics of STAD patients

\begin{tabular}{|c|c|c|}
\hline Clinical characteristics & Total & $\%$ \\
\hline Age, median (range) & $67(35-90)$ & \\
\hline \multicolumn{3}{|l|}{ Gender } \\
\hline Male & 241 & $64.27 \%$ \\
\hline Female & 134 & $35.73 \%$ \\
\hline \multicolumn{3}{|l|}{ Histologic grade } \\
\hline G1 & 10 & $2.73 \%$ \\
\hline $\mathrm{G} 2$ & 137 & $37.43 \%$ \\
\hline G3 & 219 & $59.84 \%$ \\
\hline \multicolumn{3}{|l|}{ Clinical stage } \\
\hline$\square$ & 53 & $15.06 \%$ \\
\hline ૧ & 111 & $31.53 \%$ \\
\hline$\square$ & 150 & $42.61 \%$ \\
\hline ૧ & 38 & $10.80 \%$ \\
\hline \multicolumn{3}{|l|}{$\mathrm{T}$} \\
\hline 1 & 19 & $5.18 \%$ \\
\hline 2 & 80 & $21.80 \%$ \\
\hline 3 & 168 & $45.78 \%$ \\
\hline 4 & 100 & $27.25 \%$ \\
\hline \multicolumn{3}{|l|}{$\mathrm{N}$} \\
\hline NO & 111 & $31.09 \%$ \\
\hline N1-N3 & 246 & $68.91 \%$ \\
\hline \multicolumn{3}{|l|}{ M } \\
\hline MO & 330 & $92.96 \%$ \\
\hline M1 & 25 & $7.04 \%$ \\
\hline \multicolumn{3}{|l|}{ Survival status } \\
\hline Alive & 244 & $65.07 \%$ \\
\hline Dead & 131 & $34.93 \%$ \\
\hline
\end{tabular}

\section{PDGFRB was overexpressed in STAD}

An aberrant expression of PDGFRB was observed in severe types of solid tumors. By analyzing data from TCGA, we describe the characteristics of the expression of PDGFRB in multiple types of solid tumors, including STAD (Fig. 1). In our study, the Wilcoxon rank sum test was used to compare the expression of PDGFRB in 375 STAD tissues and 32 normal samples. PDGFRB was significantly upregulated in STAD ( $P=5.956 \mathrm{e}-11)$ (Fig. 2A). Compared with 27 adjacent normal tissues, the 
expression of PDGFRB was significantly increased in STAD $(P=8.146 \mathrm{e} 囚 06)$ based on Wilcoxon signed-rank tests (Fig. 2B). In addition, we use the GEPIA database (http://gepia.cancer-pku.cn/) containing more normal samples to verify the above results, as is shown in Fig. 2C, the expression of PDGFRB is significantly higher in tumor tissues than in normal ones in STAD.

\section{The effect of PDGFRB overexpression on clinicopathological characteristics}

As shown in Figs. 3A-E, compared to the histologic grade G1-2, the expression of PDGFRB in G3 patients was significantly increased (G1-2 vs. G3, $P=4.339 \mathrm{e}-05)$. In addition, there was a significant correlation between the increased expression of $P D G F R B$ and topography (T1-2 vs. T3-4, $P=0.002$ ). With the increase in topography (from T1-T4), the expression of PDGFRB also increased $(P=8.357 \mathrm{e}-06)$. In terms of clinical stage, significant difference was observed $(P=0.007)$.

Logistic regression was used to analyse the relationship between PDGFRB expression and clinicopathological characteristics (Table 2). We found that the overexpression of $P D G F R B$ was significantly related to the clinical stage of the

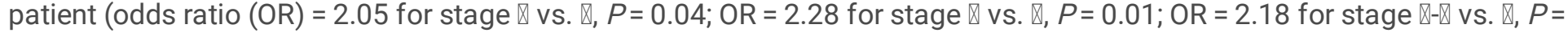
$0.01)$, primary tumour topography $(\mathrm{OR}=2.01$ for with T3-4 vs. T1-2, $P=0.004)$ and histologic grade $(\mathrm{OR}=1.95$ for with $\mathrm{G} 3$ vs. G1-2, $P=0.002$ ). Taken together, the high expression of $P D G F R B$ is closely related to worse clinicopathological characteristics than low expression.

Table 2

Relationship between PDGFRB expression and clinicopathologic characteristics by logistic regression.

\begin{tabular}{|c|c|c|c|}
\hline Clinical characteristics & Total (N) & Odds ratio in PDGFRB expression & P-value \\
\hline Age (continuous) & 371 & $1.00(0.98-1.02)$ & 0.87 \\
\hline Gender (male vs. female) & 375 & $1.14(0.75-1.74)$ & 0.54 \\
\hline Grade (G3 vs. G1-2) & 366 & $1.95(1.28-2.99)$ & 0.002 \\
\hline Distant metastasis (Yes vs. No) & 355 & $1.56(0.69-3.67)$ & 0.30 \\
\hline Lymph nodes (Yes vs. No) & 357 & $1.02(0.65-1.60)$ & 0.94 \\
\hline Stage (囚- $\nabla$ vs. $\rrbracket)$ & 352 & $2.18(1.20-4.09)$ & 0.01 \\
\hline Stage ( $₫$ vs. $\rrbracket)$ & 164 & $2.05(1.05-4.11)$ & 0.04 \\
\hline 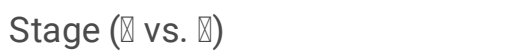 & 203 & $2.28(1.20-4.46)$ & 0.01 \\
\hline Stage ( $₫$ vs. $\rrbracket)$ & 92 & $2.16(0.93-5.14)$ & 0.08 \\
\hline Status (Dead vs. Alive) & 375 & $1.51(0.98-2.31)$ & 0.06 \\
\hline Topography (T3-4 vs. T1-2) & 367 & $2.01(1.26-3.24)$ & 0.004 \\
\hline
\end{tabular}

\section{Correlation between PDGFRB expression and survival}

Kaplan-Meier analysis revealed that increased PDGFRB expression (High vs. Low, $P=0.008$ and $P=0.025$ ) was significantly correlated with worse overall survival (OS) (Fig. 4).

For OS, univariate analysis using the Cox regression model showed that poor OS had a significant correlation with clinical

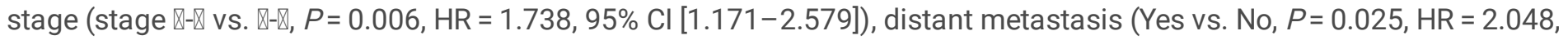
95\% CI [1.096-3.827]) and PDGFRB expression (High vs. low; $P=0.023, \mathrm{HR}=1.560,95 \% \mathrm{Cl}[1.063-2.290]$ ) (Table 3). However, in multivariate Cox regression, high PDGFRB expression (High vs. Low; $P=0.043, \mathrm{HR}=1.498,95 \% \mathrm{Cl}[1.013-$ $2.214]$ ), and distant metastasis (Yes vs. No, $P=0.026, \mathrm{HR}=2.128,95 \% \mathrm{Cl}[1.094-4.140])$ and patient age $(P=0.019, \mathrm{HR}=$ $1.585,95 \% \mathrm{Cl}[1.077-2.333])$ independently predicted adverse OS (Table 3, Fig. 5). In addition, this also revealed that STAD 
patients with upregulated PDGFRB had a 1.498-fold higher risk of unfavourable OS than patients with low expression of PDGFRB.

Table 3

Univariate and multivariate analysis of factors related to patients' overall survival.

\begin{tabular}{|c|c|c|c|c|c|c|}
\hline \multirow[t]{2}{*}{ Characteristic variable } & \multicolumn{3}{|c|}{ Univariate analysis } & \multicolumn{3}{|c|}{ Multivariate analysis } \\
\hline & HR & $95 \% \mathrm{Cl}$ & $p$-value & HR & $95 \% \mathrm{Cl}$ & $p$-value \\
\hline Age $(\geq 70$ vs. $<70)$ & 1.426 & $0.980-2.076$ & 0.064 & 1.585 & $1.077-2.333$ & 0.019 \\
\hline Gender (Male vs. Female) & 1.484 & $0.980-2.247$ & 0.062 & 1.462 & $0.959-2.228$ & 0.077 \\
\hline Grade (G3 vs. G1-2) & 1.350 & $0.910-2.004$ & 0.136 & & & \\
\hline Stage $(\rrbracket-\bigotimes$ vs. $\rrbracket-\rrbracket)$ & 1.738 & $1.171-2.579$ & 0.006 & 1.418 & $0.766-2.627$ & 0.266 \\
\hline Topography (T3-4 vs. T1-2) & 1.585 & $0.990-2.539$ & 0.055 & 1.178 & $0.681-2.036$ & 0.558 \\
\hline Distant metastasis (Yes vs. No) & 2.048 & $1.096-3.827$ & 0.025 & 2.128 & $1.094-4.140$ & 0.026 \\
\hline Lymph nodes (Yes vs. No) & 1.507 & $0.965-2.354$ & 0.071 & 1.070 & $0.570-2.008$ & 0.833 \\
\hline PDGFRB (High vs Low) & 1.560 & $1.063-2.290$ & 0.023 & 1.498 & $1.013-2.214$ & 0.043 \\
\hline
\end{tabular}

PDGFRB-related signalling pathways were analysed using GSEA

We used GSEA to screen for significantly activated signalling pathways between the high expression and low expression $P D G F R B$ phenotype groups. FDR $<0.05$ and NOM $P$-val $<0.05$ indicate significant differences in enrichment of the MSigDB collection (c2.cp.biocarta.v7 .4.symbols.gmt). We selected the top 5 most highly enriched signalling pathways in high expression phenotype group and all 9 enriched signalling pathways in low expression phenotype group based on NES, FDR and NOM $P$ values (Fig. 6 and Table 4). 
Gene sets enriched in phenotype high.

\begin{tabular}{|c|c|c|c|c|}
\hline MSigDB collection & Gene set name & NES & $\begin{array}{l}\text { NOM } \\
\text { p-val }\end{array}$ & $\begin{array}{l}\text { FDR } \\
\text { q-val }\end{array}$ \\
\hline \multirow{14}{*}{$\begin{array}{l}\text { c2.cp.biocarta.v7.4.symbols.gmt } \\
\text { (Curated) }\end{array}$} & KEGG_CYTOKINE_CYTOKINE_RECEPTOR_INTERACTION & 2.505 & 0.000 & 0.000 \\
\hline & KEGG_FOCAL_ADHESION & 2.504 & 0.000 & 0.000 \\
\hline & KEGG_DILATED_CARDIOMYOPATHY & 2.435 & 0.000 & 0.000 \\
\hline & KEGG_ECM_RECEPTOR_INTERACTION & 2.426 & 0.000 & 0.000 \\
\hline & KEGG_HYPERTROPHIC_CARDIOMYOPATHY_HCM & 2.395 & 0.000 & 0.000 \\
\hline & KEGG_PARKINSONS_DISEASE & -2.163 & 0.000 & 0.003 \\
\hline & KEGG_OXIDATIVE_PHOSPHORYLATION & -2.189 & 0.000 & 0.003 \\
\hline & KEGG_ALZHEIMERS_DISEASE & -2.134 & 0.000 & 0.003 \\
\hline & KEGG_PROTEASOME & -2.100 & 0.000 & 0.004 \\
\hline & KEGG_HUNTINGTONS_DISEASE & -2.210 & 0.000 & 0.004 \\
\hline & KEGG_RIBOSOME & -1.980 & 0.004 & 0.014 \\
\hline & KEGG_TERPENOID_BACKBONE_BIOSYNTHESIS & -1.896 & 0.006 & 0.026 \\
\hline & KEGG_CARDIAC_MUSCLE_CONTRACTION & -1.904 & 0.000 & 0.028 \\
\hline & KEGG_SPLICEOSOME & -1.835 & 0.019 & 0.042 \\
\hline
\end{tabular}

Abbreviation: FDR, false discovery rate; NES: normalized enrichment score; NOM: nominal. Gene sets with NOM pval<0.05 and FDR q-val<0.05 are considered as significant.

Prediction of DEmiRNAs targeted by mRNA

miRNAs interacting with the target gene PDGFRB were predicted through the ENCORI database (https://rna.sysu.edu.cn), as is shown in Fig. 7. Based on the value of cor, $p$-value and logFC, hsa-miR-30c-5p, hsa-miR-30e-5p and hsa-miR-486-5p were selected for the subsequent analysis (Supplementary Table 1). As is shown in Fig. 8, the expression of the above three miRNAs was negatively correlated with that of $P D G F R B$, and $P<0.05$. Kaplan-Meier survival curve and log-rank analysis were used to evaluate the correlation between the expression of miRNAs and the prognosis of patients with STAD. A low expression of hsa-mir-30c-5p $(P=0.048)$ and hsa-mir-30e-5p $(P=0.028)$ was significantly associated with a worse OS (Fig. 9).

\section{Prediction of DElncRNAs targeted by miRNA}

According to the prognostic value of miRNAs, we finally selected hsa-mir-30e-5p as the target miRNA to predict the IncRNAs interacting with it through the same method as that used to predict DEmiRNAs. The expression of 4 IncRNAs (NORAD, CASC15, FTX and FLJ42393) had a negative correlation with that of hsa-miR-30e-5p $(P<0.05$, Supplementary Table 2, Fig. 10). The correlations between the expression of 4 IncRNAs and target gene PDGFRB were also further evaluated. Except IncRNA FLJ42393, the other 3 IncRNAs showed positive correlations with the expression of $P D G F R B(P<0.05$, Fig. 11). Meanwhile, the expression of the 4 IncRNAs was significantly higher than that under normal control (Fig. 12). However, through Kaplan-Meier survival curve and log-rank analysis, we found that only IncRNA CASC15 was significantly associated with OS among the 4 IncRNAs; patients with an elevated expression of IncRNA CASC15 tended to exhibit a poorer OS ( $P=$ 0.013 , Fig. 13). Therefore, according to the above results, a IncRNAs-miRNA-mRNA ceRNA network was constructed by using Cytoscape (Version 3.9.0), which was composed of 4 IncRNA nodes, 1 miRNA node and 1 mRNA node (Fig. 14). 


\section{Relationship between PDGFRB expression and tumor-infiltrating immune cells}

The level of tumor-infiltrating immune cells plays a key role in the prediction of OS rate and the application of immunotherapy. To verify the correlation between the expression of PDGFRB and immune infiltration, we used TIMER 2.0 database to analyze possible relations. As is shown in Fig. 15, we found that level of 5 immune cells (CD8 + T cells, CD4 + T cells, macrophages, neutrophils and dendritic cells) had positive correlations with the expression of PDGFRB in STAD. Therefore, we held the view that PDGFRB was significantly associated with the immune infiltration of several immune cells indeed.

\section{The value of PDGFRB in immunotherapy}

As the expression of $P D G F R B$ was significantly correlated with immune cell infiltration, we were curious about the function of PDGFRB in immunotherapy. Therefore, we performed a correlation analysis to investigate the correlation between the expression of PDGFRB and each immune regulator through TIMER 2.0. Immunotherapy-associated molecules PDCD1, PDCD1LG2, CTLA4, CD274, ENTPD1 and KLRC1 were regarded to have a strong correlation with the expression of PDGFRB (Fig. 16A). Besides, to verify the results from TIMER 2.0, we also investigated their association through GEPIA for conform, which demonstrated that PDGFRB had strong significant relationships with these 6 genes (PDCD1, PDCD1LG2, CTLA4, CD274, ENTPD1 and KLRC1) (Fig. 16B).

\section{Experimental verification}

We used IHC technology to check the expression level of PDGFRB, CD4, CD8, PD-1, PD-L1 and CTLA-4 in normal and carcinoma tissue. Compared to normal control, it existed a large amount of CD4 + and CD8 + T lymphocytes infiltration in tumor microenvironment. The expression of immune checkpoint molecules PD-1, PD-L1 and CTLA-4 also significantly increased, even though the difference of PD-1 expression between normal and carcinoma tissue did not reach statistical significance. In carcinoma tissue, the expression of PDGFRB by tumor-associated stromal cells was obviously elevated than normal tissue (Figs. 17 and 18).

Our study also verified the existence of positive correlations between expression level of PDGFRB and immune checkpoint molecules (PD-L1 and CTLA-4) in gastric carcinoma, though significant difference was not reached between PDGFRB and PD-1 (Fig. 19).

\section{Discussion}

Gastric cancer is a malignant tumour originating from the epithelium of the gastric mucosa, accompanied by a highly aggressive and heterogeneous nature. Gastric cancer is also a multifactorial disease. Environmental factors and genetic factors have an important influence on the occurrence and development of gastric cancer. A high-fat, high-salt and highnitrogen diet, Helicobacter pylori, Epstein-Barr virus infection history, precancerous gastric lesions and smoking have been reported as risk factors for gastric tumorigenesis (Yusefi et al. 2018). With the improvement of eating habits and the development of advanced diagnosis and treatment strategies, the morbidity and mortality of gastric cancer have decreased significantly in the past few decades. The five-year survival rate of early gastric cancer can reach $90 \%$. Due to the very low early diagnosis rate, many patients miss the best treatment opportunity and progress to advanced disease (Tan 2019). Advanced gastric cancer tends to have a more severe prognosis, with a median survival period of fewer than 12 months (Machlowska et al. 2020; Zhang and Zhang 2017). To solve this challenge, finding effective therapeutic targets and potential prognostic markers is very important in individualized treatment.

The PDGFRB gene is located on chromosome $5 q 31-q 32$, through which tyrosine kinase receptors binding PDGFB and PDGFD are encoded and highly expressed in fibroblasts and pericytes. Recently, it is reported in several researches that mutations of PDGFRB were identified in a few patients with hereditary and sporadic infantile myofibromatosis (Arts et al. 2016; Arts et al. 2017; Cheung et al. 2013). Zhaoming Li et al. (Li et al. 2019) found that PDGFRB mutations encoding 
p.Asn666Ser constitutively activated PDGFRB and the mutations might play a critical role in the pathogenesis of unicentric Castleman disease. Therefore, the gain-of-function mutations of PDGFRB may be sensitive to tyrosine kinase inhibitors. In addition, a high expression of PDGFRB is related to high grades, tumor progression and poorer clinical outcomes in patients with human breast cancer (Primac et al. 2019), gastric cancer (Wang et al. 2019; Wang et al. 2017), glioblastoma (Xu and Li 2016) and colorectal cancer (Steller et al. 2013) involving a variety of different signaling pathways. PDGFRB might be a potential therapeutic target for cancers and other diseases.

In recent years, the emergence of bioinformatics has attracted much attention and created breakthroughs in the search for protooncogenes. With the help of bioinformatics, various molecular subtypes, biomarkers of prognostic predictions, new targeted drug development and applications have been identified (Wen et al. 2018; Xu et al. 2016; Zhou et al. 2018). In our study, according to the analysis of the TCGA data, we found that the expression level of PDGFRB in tumour samples was significantly higher than that in the normal group. Highly expressed PDGFRB is obviously related to poorly differentiated histologic grade and high topography. Patients with elevated expression of PDGFRB have significantly worse OS. Multivariate analysis showed that high PDGFRB expression is an independent prognostic factor for OS, which is consistent with the study of Uthandaraman Mahalinga Raja (Raja et al. 2017).

Through GSEA, we screened out signalling pathways, such as FOCAL-ADHESION based on NES, FDR and NOM $P$ va/ were differentially enriched in the high PDGFRB expression phenotype, and nine signaling pathways such as PARKINSONSDISEASE were significantly enriched in the low PDGFRB expression phenotype. Focal adhesion kinase (FAK) is a nonreceptor tyrosine kinase that plays a key role in the regulation of cell adhesion, migration, proliferation and survival. In cancer, FAK is a major driver of invasion and metastasis, and its upregulation is associated with poor patient prognosis (Tapial Martínez et al. 2020). The correlation between PDGFRB and the enriched pathways in the high expression phenotype of PDGFRB remain unclear in gastric cancer. The interaction between them needs to be further explored and studied in the future.

Recently, emerging evidence has shown that noncoding RNAs, including microRNAs, long noncoding RNAs and circular RNAs play a key role in various biological processes, such as cell proliferation and apoptosis (Santosh et al. 2015; Zhu et al. 2019). miRNAs function by binding the complementary 3 '- untranslated region (UTR) with their target mRNAs, which leads to either post transcriptional inhibition of the target gene or mRNA degradation. One hypothesis was proposed on ceRNA by Salmena et al. in 2011 (Salmena et al. 2011). A complicated post transcriptional regulatory network was described, that is, IncRNAs, circular RNAs, mRNAs and other RNAs competed with miRNAs via serving as natural miRNA sponges by miRNA response elements (MRE) to regulate the expression level of mRNAs (Long et al. 2019). Recently, it has been verified in an increasing number of studies that the IncRNA-miRNA-mRNA regulatory network plays a critical role in the progression and pathogenesis of several tumors, including hepatocarcinogenesis, esophageal cancer, colorectal cancer, and other malignant tumors (Ghasemi et al. 2020; Liu et al. 2020; Zhang et al. 2020) through multiple levels of regulation, which involve transcriptional, post transcriptional and epigenetic regulation.

In our study, 1 IncRNA (casc15), 2 miRNAs (has-mir-30c-5p and has-mir-30e-5p) and the mRNA (PDGFRB) were identified as potential biomarkers for the prognosis of patients with STAD via a survival analysis. It has been reported in several literatures that overexpressed LncRNA CASC15 was involved in several tumor types. S Shan et al. (Shan et al. 2019) found that an elevated LnCRNA CASC15 was associated with tumor growth and predicted a poorer prognosis in cervical cancer. Niancai Jing et al. (Jing et al. 2018) found that the knockdown of CASC15 significantly inhibited the proliferation, migration and invasion of colon cancer cells in vitro and in vivo by inactivating Wnt/ $\beta$-catenin signaling pathway in a miR-4310/LGR5dependent manner in colorectal cancer. Zhibin Zuo et al. (Zuo et al. 2018) found that an overexpressed IncRNA CASC15 promoted the progression of tongue squamous cell carcinoma through targeting mir-33a-5p in basic research. Besides, our study also showed that the expression of IncRNA CASC15 was higher in STAD tissues than in paired non-tumor tissues. An increased expression of IncRNA CASC15 is associated with a worse prognosis in patients with STAD. We found that IncRNA CASC15 might compete with one key DEmiRNA (has-miR-30e-5p) to mediate the expression of PDGFRB in gastric cancer according to bioinformatics analysis. Therefore, the IncRNA CASC15-has-miR-30e-5p-PDGFRB axis may regulate biological 
processes and pathways of STAD and provide potential therapeutic strategies for gastric cancer. However, in ovarian cancer, Yin Shi (Shi et al. 2019) observed an opposite result that a low expression of CASC15 was closely associated with an advanced TNM stage, a moderate/poor differentiation, and a larger tumor size. Moreover, Kaplan-Meier survival analysis showed that patients with a low expression of CASC15 presented poorer overall survival and progression-free survival than those with a higher expression of CASC15. Thus, comprehensive and systematic genetic-level profiling was also vital.

Equally important, as immune cells play a pivotal role in predicting the prognosis of patients in tumor microenvironment (Huang et al. 2021), we used TIMER 2.0 database to find correlations among the expression of PDGFRB, immune infiltration and the value of $P D G F R B$ in immunotherapy. We found that the associations of PDGFRB with CD $8+\mathrm{T}$ cells, CD $4+\mathrm{T}$ cells, $\mathrm{B}$ cells, macrophages, neutrophils and dendritic cells were strong. An excess level of PDGFRB in STAD can trigger anti-tumor immune responses. Together, these findings indicate that PDGFRB plays a key role in the recruitment and regulation of immune infiltrating cells in STAD. Therefore, we considered PDGFRB as a biomarker of immune infiltration and further evaluated its function in immunotherapy. TIMER2.0 database and GEPIA database both showed significant correlations between an elevated PDGFRB and immunotherapeutic targets PDCD1, PDCD1LG2, CTLA4, CD274, ENTPD1 as well as $K L R C 1$. In addition, we also proved the positive relationships between the expression of PD-L1, CTLA-4 and PDGFRB through IHC experiment. Based on the above results, we speculate that through IncRNA CASC15-has-miR-30e-5p-PDGFRB axis-based treatment strategies combined with immunotherapy, more beneficial effects may be able to be gained than just using a single remedy in STAD with an overexpressed PDGFRB.

In conclusion, patients with an elevated PDGFRB and IncRNA CASC15 together with a decreased has-miR-30e-5p have a poor prognosis, and an increased PDGFRB may serve as a promising and potential independent prognostic biomarker of a poor survival and therapeutic targets in STAD. We constructed a IncRNA CASC15-has-miR-30e-5p-PDGFRB axis for the first time through bioinformatics methods, which also provided novel insights into the pathogenesis, progression and prognosis of STAD. The value of PDGFRB in immunotherapy evaluated by GEPIA and TIMER 2.0 database showed that STAD with an overexpressed PDGFRB might benefit from immune checkpoint inhibitors. Further experimental research is needed to verify this underlying biological regulatory mechanism and therapeutic insights.

\section{Methods}

\section{Patient information}

This study was performed according to institutional review board (IRB) requirement of Mudanjiang Medical University (2021-MYWZ42). We collected 12 surgical resected gastric adenocarcinoma and 4 normal control samples at Mudanjiang Medical University affiliated hospital. Formalin-fixed paraffin-embedded (FFPE) tissue blocks from patients were obtained. Informed consent was obtained by the opt-out method. It was approved by the ethics committee of Mudanjiang Medical University.

RNA-sequencing data, miRNA sequencing data and corresponding patient clinical information were collected from the TCGA database (https://portal.gdc.cancer.gov/repository), containing 375 STAD samples and 32 normal samples. Workflow type was HTSeq-FPKM. The clinical characteristics of the STAD patients, including their age, sex, histologic grade, clinical stage, primary tumour $\mathrm{T}$, lymph node $\mathrm{N}$, metastasis $\mathrm{M}$, and survival status, were recorded, as shown in Table 1. Some unavailable or unclear clinical data were deleted.

\section{MultiGSEA}

Gene set enrichment analysis (GSEA) is a computational method to determine whether a previously defined genome has consistent statistically significant differences in two biological states (Jin et al. 2020). GSEA was carried out to elucidate the significant survival difference observed between the high- and low-PDGFRB groups. The expression level of PDGFRB served 
as a phenotype label. The gene set permutation number was 1000 times for each analysis. The statistical significance of the pathways depended on a normal $P$ value less than 0.05 and a false discovery rate (FDR) q-val less than 0.05 .

\section{miRNA and IncRNA expression profiling}

ENCORI database (https://rna.sysu.edu.cn) was applied to screen out miRNAs and IncRNAs interacting with the target gene. miRNAs identified by at least two out of 7 relevant databases were considered as candidate miRNAs, whose correlations with the target gene and IncRNAs were analyzed in R language. The differentially expressed miRNAs (DEmiRNAs) and differentially expressed IncRNAs (DElncRNAs) in gastric cancer and normal tissues were screened out individually through the edgeR package in the $\mathrm{R}$ language.

\section{Construction of the ceRNA axis}

A IncRNA-miRNA-mRNA ceRNA network was constructed dependent on the DEmiRNA- DEIncRNA and DEmiRNA-mRNA interactions. Cytoscape (Version 3.9.0) was utilized to visualize the ceRNA network.

\section{Survival analysis}

For all DEmiRNAs, DElncRNAs and mRNA in the ceRNA network, patients with stomach adenocarcinoma were classified into high- and low-expression group based on the median value of specific RNAs. The differences between the overall survival of high- and low-expression group were evaluated by using the survival package in $\mathrm{R}$ language. A $P$ value $\mathbb{0} 0.05$ indicated statistical significance.

\section{Tumour-infiltrating immune cell analysis}

The TIMER 2.0 (http://timer.cistrome.org/) was used to access the potential correlation between the expression of PDGFRB and tumor-infiltrating immune cells, including CD8+ T cells, CD4+ T cells, B cells, macrophages, neutrophils and dendritic cells. The relationship among immunotherapy-related targets, such as PDCD1, PDCD1LG2, CTLA4, CD274, ENTPD1, $K L R C 1$ and the target gene were also evaluated based on both the TIMER 2.0 (http://timer.cistrome.org/) and GEPIA database (http://gepia.cancer-pku.cn/).

\section{Immunohistochemistry}

FFPE tissue blocks were cut into $4 \mu \mathrm{m}$-thick tissue sections and subjected to both hematoxylin and eosin staining and immunohistochemical staining. For IHC, an automated Bond-III slide stainer (Leica Biosystems, Wetzlar, Germany) was used to detect following antibodies against CD8 (clone: C8/144B, 1:200, Nichirei), CD4 (clone: 1F6, 1:50, Nichirei ), PD-1 (clone: NAT, 1:100, Abcom), PD-L1 (clone SP263; rabbit monoclonal primary anti-PD-L1 antibody, prediluted, Ventana Medical Systems, Tucson, AZ) , CTLA-4 (clone: F-8, 1:200, Santa Cruz) and PDGFRB (clone: D-6, 1:200, Santa Cruz) in accordance with the manufacturer's protocol. Image $J$ was applied for the evaluation of percentage of positive cells or positive regions.

\section{Statistical analysis}

All statistical analyses were performed using R (version 3.6.1, 2019-07-05, R Foundation, Vienna, Austria) and GraphPad Prism 9. The expression of PDGFRB and IncRNAs were compared between the STAD and normal groups by Wilcoxon rank sum tests, respectively. The expression of PDGFRB, CD4, CD8, PD-1, PD-L1 and CTLA-4 between STAD and normal control was compared by the nonpaired Student's $t$-test. The correlation between PDGFRB expression and clinicopathological characteristics was performed using the Wilcoxon signed-rank test and logistic regression. In the Cox regression analysis, a $P$ value $\otimes 0.05$ indicated statistical significance. The median expression value of PDGFRB was considered as a cut-off value. Spearman rank-based correlation coefficients were used to estimate the correlation between two continuous variables, and corresponding P-values were calculated. 


\section{Declarations}

\section{Author contributions}

GY and YRZ had the idea for the article and performed the literature search and data analysis; GY and SZJ drafted; BY critically revised the work; YW performed IHC diagnosis and analysis. All authors reviewed the manuscript

\section{Data availability statement}

All data generated or analyzed during this study are included in this article and its Supplementary Material. All data associated with the paper is available and it can be accessed by contacting the corresponding author Bin Yan.

\section{Additional information}

The authors have no relevant financial or non-financial interests to disclose

\section{Ethics approval}

This study was performed in accordance with the principle of the Helsinki Declaration and approved by the Ethics Committee of Mudanjiang Medical University Affiliated Hospital (Ethics 2021-MYWZ42).

\section{References}

1. Wang, H., Shen, L., Li, Y. \& Lv, J. Integrated characterisation of cancer genes identifies key molecular biomarkers in stomach adenocarcinoma. Journal of clinical pathology 73, 579-586, doi:10.1136/jclinpath-2019-206400 (2020).

2. Johnston, F. M. \& Beckman, M. Updates on Management of Gastric Cancer. Current oncology reports 21, 67, doi:10.1007/s11912-019-0820-4 (2019).

3. Machlowska, J., Baj, J., Sitarz, M., Maciejewski, R. \& Sitarz, R. Gastric Cancer: Epidemiology, Risk Factors, Classification, Genomic Characteristics and Treatment Strategies. International journal of molecular sciences 21, doi:10.3390/ijms21114012 (2020).

4. Sitarz, R. et al. Gastric cancer: epidemiology, prevention, classification, and treatment. Cancer management and research 10, 239-248, doi:10.2147/cmar.S149619 (2018).

5. Petryszyn, P., Chapelle, N. \& Matysiak-Budnik, T. Gastric Cancer: Where Are We Heading? Digestive diseases (Basel, Switzerland) 38, 280-285, doi:10.1159/000506509 (2020).

6. Sexton, R. E., Al Hallak, M. N., Diab, M. \& Azmi, A. S. Gastric cancer: a comprehensive review of current and future treatment strategies. Cancer metastasis reviews 39, 1179-1203, doi:10.1007/s10555-020-09925-3 (2020).

7. Kim, H. J. et al. Genetic association between 5'-upstream single-nucleotide polymorphisms of PDGFRB and schizophrenia in a Korean population. Schizophrenia research 103, 201-208, doi:10.1016/j.schres.2008.04.031 (2008).

8. Dachy, G. et al. Association of PDGFRB Mutations With Pediatric Myofibroma and Myofibromatosis. JAMA dermatology 155, 946-950, doi:10.1001/jamadermatol.2019.0114 (2019).

9. Mathorne, S. W., Sørensen, K., Fagerberg, C., Bode, M. \& Hertz, J. M. A novel PDGFRB sequence variant in a family with a mild form of primary familial brain calcification: a case report and a review of the literature. BMC neurology 19, 60, doi:10.1186/s12883-019-1292-8 (2019).

10. Li, Z. et al. Recurrent PDGFRB mutations in unicentric Castleman disease. Leukemia 33, 1035-1038, doi:10.1038/s41375-018-0323-6 (2019).

11. Karasozen, Y. et al. Somatic PDGFRB Activating Variants in Fusiform Cerebral Aneurysms. American journal of human genetics 104, 968-976, doi:10.1016/j.ajhg.2019.03.014 (2019). 
12. Hassan, M. et al. Novel PDGFRB rearrangement in multifocal infantile myofibromatosis is tumorigenic and sensitive to imatinib. Cold Spring Harbor molecular case studies 5, doi:10.1101/mcs.a004440 (2019).

13. Lecerf, C. et al. Immune gene expression in head and neck squamous cell carcinoma patients. European journal of cancer (Oxford, England: 1990) 121, 210-223, doi:10.1016/j.ejca.2019.08.028 (2019).

14. Zhang, N. et al. The overexpression of PDGF-BB and its receptor is correlated with lymphatic metastasis in patients with non-small cell lung cancer. International journal of clinical and experimental pathology 11, 6010-6017 (2018).

15. Salmena, L., Poliseno, L., Tay, Y., Kats, L. \& Pandolfi, P. P. A ceRNA hypothesis: the Rosetta Stone of a hidden RNA language? Cell 146, 353-358, doi:10.1016/j.cell.2011.07.014 (2011).

16. Fan, Q. \& Liu, B. Comprehensive analysis of a long noncoding RNA-associated competing endogenous RNA network in colorectal cancer. OncoTargets and therapy 11, 2453-2466, doi:10.2147/ott.S158309 (2018).

17. Mendell, J. T. Targeting a Long Noncoding RNA in Breast Cancer. The New England journal of medicine 374, 22872289, doi:10.1056/NEJMcibr1603785 (2016).

18. Quinn, J. J. \& Chang, H. Y. Unique features of long non-coding RNA biogenesis and function. Nature reviews. Genetics 17, 47-62, doi:10.1038/nrg.2015.10 (2016).

19. Kornienko, A. E., Guenzl, P. M., Barlow, D. P. \& Pauler, F. M. Gene regulation by the act of long non-coding RNA transcription. BMC biology 11, 59, doi:10.1186/1741-7007-11-59 (2013).

20. Shi, X., Sun, M., Liu, H., Yao, Y. \& Song, Y. Long non-coding RNAs: a new frontier in the study of human diseases. Cancer letters 339, 159-166, doi:10.1016/j.canlet.2013.06.013 (2013).

21. Mercer, T. R. \& Mattick, J. S. Structure and function of long noncoding RNAs in epigenetic regulation. Nature structural \& molecular biology 20, 300-307, doi:10.1038/nsmb.2480 (2013).

22. Yin, J. et al. Construction and analysis of a IncRNA-miRNA-mRNA network based on competitive endogenous RNA reveal functional IncRNAs in oral cancer. BMC medical genomics 13, 84, doi:10.1186/s12920-020-00741-w (2020).

23. Chen, Z. et al. IncRNA DSCR8 mediates miR-137/Cdc42 to regulate gastric cancer cell proliferation, invasion, and cell cycle as a competitive endogenous RNA. Molecular therapy oncolytics 22, 468-482, doi:10.1016/j.omto.2021.05.010 (2021).

24. Jin, C. Y. et al. High expression of RRM2 as an independent predictive factor of poor prognosis in patients with lung adenocarcinoma. Aging 13, 3518-3535, doi:10.18632/aging.202292 (2020).

25. Yusefi, A. R., Bagheri Lankarani, K., Bastani, P., Radinmanesh, M. \& Kavosi, Z. Risk Factors for Gastric Cancer: A Systematic Review. Asian Pacific journal of cancer prevention: APJCP 19, 591-603, doi:10.22034/apjcp.2018.19.3.591 (2018).

26. Tan, Z. Recent Advances in the Surgical Treatment of Advanced Gastric Cancer: A Review. Medical science monitor: international medical journal of experimental and clinical research 25, 3537-3541, doi:10.12659/msm.916475 (2019).

27. Zhang, X. Y. \& Zhang, P. Y. Gastric cancer: somatic genetics as a guide to therapy. Journal of medical genetics $54,305-$ 312, doi:10.1136/jmedgenet-2016-104171 (2017).

28. Cheung, Y. H. et al. A recurrent PDGFRB mutation causes familial infantile myofibromatosis. American journal of human genetics 92, 996-1000, doi:10.1016/j.ajhg.2013.04.026 (2013).

29. Arts, F. A. et al. PDGFRB mutants found in patients with familial infantile myofibromatosis or overgrowth syndrome are oncogenic and sensitive to imatinib. Oncogene 35, 3239-3248, doi:10.1038/onc.2015.383 (2016).

30. Arts, F. A. et al. PDGFRB gain-of-function mutations in sporadic infantile myofibromatosis. Human molecular genetics 26, 1801-1810, doi:10.1093/hmg/ddx081 (2017).

31. Primac, I. et al. Stromal integrin a11 regulates PDGFR- $\beta$ signaling and promotes breast cancer progression. The Journal of clinical investigation 129, 4609-4628, doi:10.1172/jci125890 (2019).

32. Wang, J. X. et al. GLI2 induces PDGFRB expression and modulates cancer stem cell properties of gastric cancer. European review for medical and pharmacological sciences 21, 3857-3865 (2017). 
33. Wang, G. et al. Hypomethylated gene NRP1 is co-expressed with PDGFRB and associated with poor overall survival in gastric cancer patients. Biomedicine \& pharmacotherapy = Biomedecine \& pharmacotherapie 111, 1334-1341, doi:10.1016/j.biopha.2019.01.023 (2019).

34. Xu, G. \& Li, J. Y. Differential expression of PDGFRB and EGFR in microvascular proliferation in glioblastoma. Tumour biology: the journal of the International Society for Oncodevelopmental Biology and Medicine 37, 10577-10586, doi:10.1007/s13277-016-4968-3 (2016).

35. Steller, E. J. et al. PDGFRB promotes liver metastasis formation of mesenchymal-like colorectal tumor cells. Neoplasia (New York, N.Y.) 15, 204-217, doi:10.1593/neo.121726 (2013).

36. Wen, P. et al. Identification of candidate biomarkers and pathways associated with SCLC by bioinformatics analysis. Molecular medicine reports 18, 1538-1550, doi:10.3892/mmr.2018.9095 (2018).

37. Xu, Z. et al. Identification of candidate biomarkers and analysis of prognostic values in ovarian cancer by integrated bioinformatics analysis. Medical oncology (Northwood, London, England) 33, 130, doi:10.1007/s12032-016-0840-y (2016).

38. Zhou, L. et al. Bioinformatics analyses of significant genes, related pathways and candidate prognostic biomarkers in glioblastoma. Molecular medicine reports 18, 4185-4196, doi:10.3892/mmr.2018.9411 (2018).

39. Raja, U. M., Gopal, G., Shirley, S., Ramakrishnan, A. S. \& Rajkumar, T. Immunohistochemical expression and localization of cytokines/chemokines/growth factors in gastric cancer. Cytokine 89, 82-90, doi:10.1016/j.cyto.2016.08.032 (2017).

40. Tapial Martínez, P., López Navajas, P. \& Lietha, D. FAK Structure and Regulation by Membrane Interactions and Force in Focal Adhesions. Biomolecules 10, doi:10.3390/biom10020179 (2020).

41. Zhu, J. et al. IncRNA/circRNA-miRNA-mRNA ceRNA network in lumbar intervertebral disc degeneration. Molecular medicine reports 20,3160-3174, doi:10.3892/mmr.2019.10569 (2019).

42. Santosh, B., Varshney, A. \& Yadava, P. K. Non-coding RNAs: biological functions and applications. Cell biochemistry and function 33, 14-22, doi:10.1002/cbf.3079 (2015).

43. Long, J. et al. Construction and comprehensive analysis of a ceRNA network to reveal potential prognostic biomarkers for hepatocellular carcinoma. Cancer cell international 19, 90, doi:10.1186/s12935-019-0817-y (2019).

44. Zhang, R. et al. Candidate IncRNA-miRNA-mRNA network in predicting hepatocarcinogenesis with cirrhosis: an integrated bioinformatics analysis. Journal of cancer research and clinical oncology 146, 87-96, doi:10.1007/s00432019-03090-z (2020).

45. Liu, H. et al. Differential Analysis of IncRNA, miRNA and mRNA Expression Profiles and the Prognostic Value of IncRNA in Esophageal Cancer. Pathology oncology research: POR 26, 1029-1039, doi:10.1007/s12253-019-00655-8 (2020).

46. Ghasemi, T., Khalaj-Kondori, M., Hosseinpour Feizi, M. A. \& Asadi, P. IncRNA-miRNA-mRNA interaction network for colorectal cancer; An in silico analysis. Computational biology and chemistry 89, 107370, doi:10.1016/j.compbiolchem.2020.107370 (2020).

47. Shan, S. et al. Higher IncRNA CASC15 expression predicts poor prognosis and associates with tumor growth in cervical cancer. European review for medical and pharmacological sciences 23, 507-512, doi:10.26355/eurrev_201901_16862 (2019).

48. Jing, N. et al. LncRNA CASC15 promotes colon cancer cell proliferation and metastasis by regulating the miR4310/LGR5/Wnt/ $\beta$-catenin signaling pathway. Molecular medicine reports 18, 2269-2276, doi:10.3892/mmr.2018.9191 (2018).

49. Zuo, Z., Ma, L., Gong, Z., Xue, L. \& Wang, Q. Long non-coding RNA CASC15 promotes tongue squamous carcinoma progression through targeting miR-33a-5p. Environmental science and pollution research international 25, 2220522212, doi:10.1007/s11356-018-2300-z (2018).

50. Shi, Y., Gao, S., Zheng, Y., Yao, M. \& Ruan, F. LncRNA CASC15 Functions As An Unfavorable Predictor Of Ovarian Cancer Prognosis And Inhibits Tumor Progression Through Regulation Of miR-221/ARID1A Axis. OncoTargets and therapy 12 ,

Page $14 / 28$ 
8725-8736, doi:10.2147/ott.S219900 (2019).

51. Huang, X. Y., Liu, J. J., Liu, X., Wang, Y. H. \& Xiang, W. Bioinformatics analysis of the prognosis and biological significance of VCAN in gastric cancer. Immunity, inflammation and disease 9, 547-559, doi:10.1002/iid3.414 (2021).

\section{Figures}

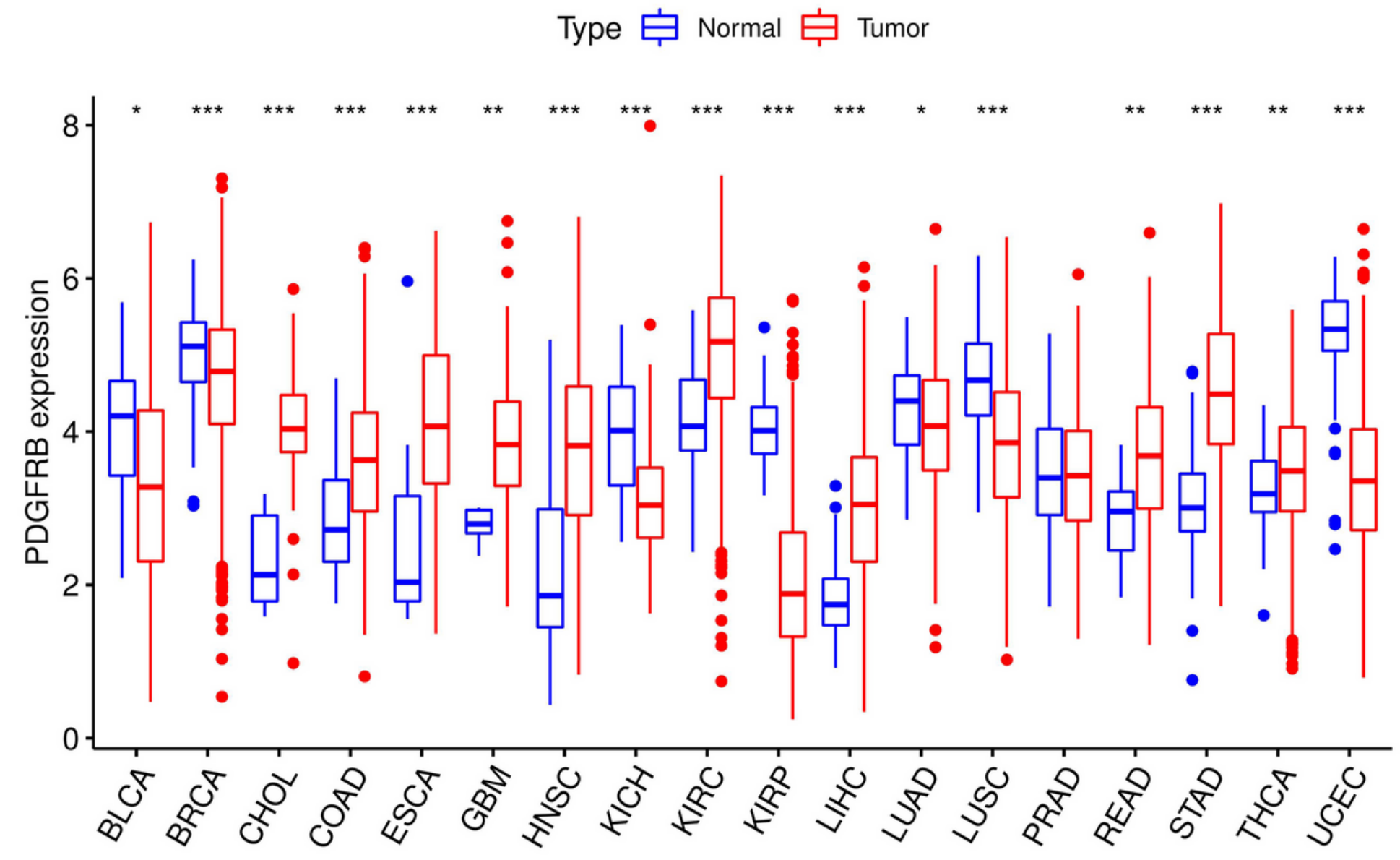

Figure 1

The expression status at mRNA level of the PDGFRB gene in different cancers. ${ }^{\star} \mathrm{P}<0.05 ; * \star \mathrm{P}<0.01 ; * \star \star \mathrm{P}<0.001$. 

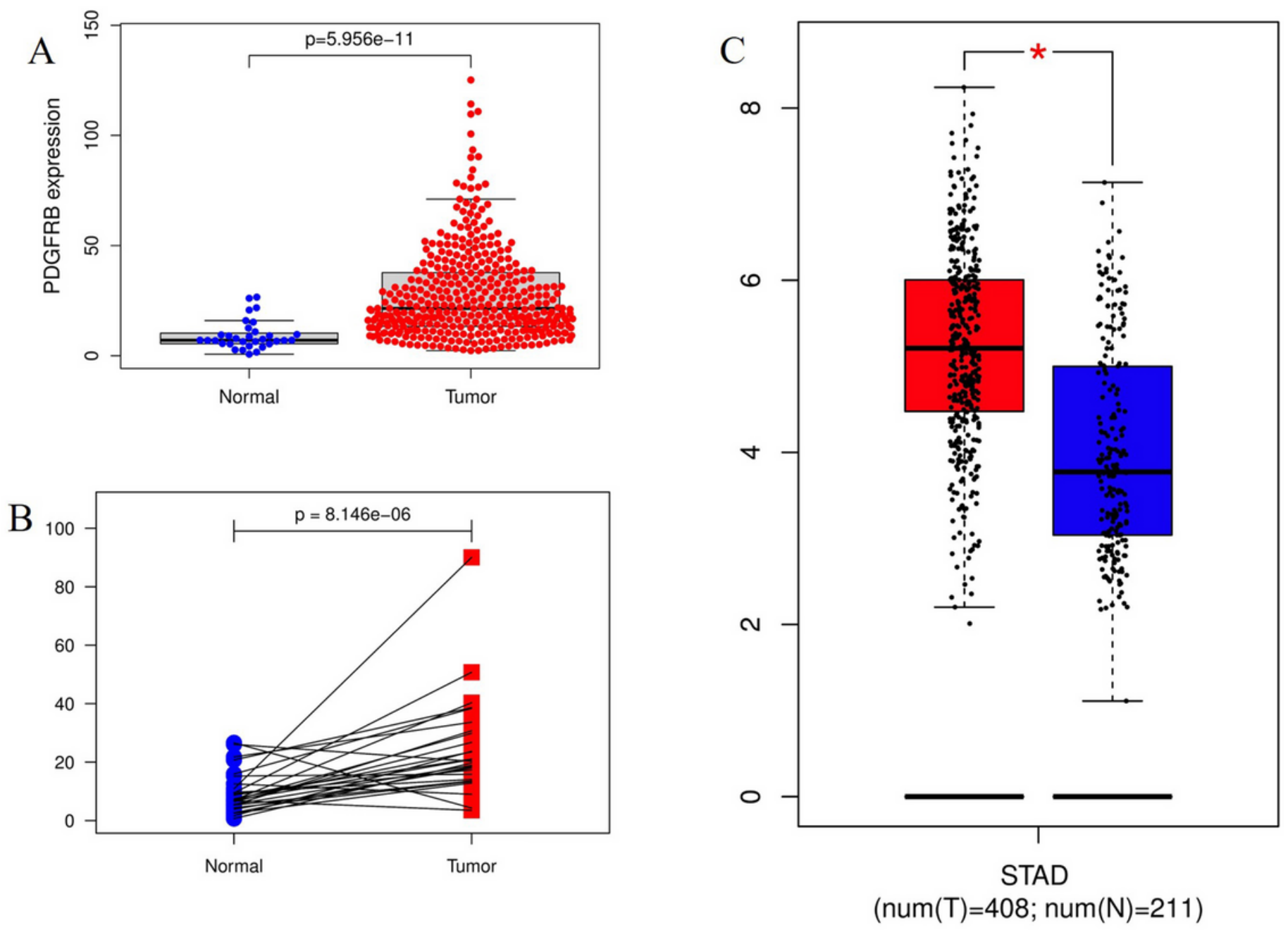

Figure 2

The expression of PDGFRB was increased in STAD. (A) PDGFRB showed significantly higher expression in STAD samples than in normal samples through the Wilcoxon rank sum test. (B) The expression of PDGFRB was significantly increased in STAD tissues compared with adjacent noncancerous tissues through the Wilcoxon signed rank test. (C) The expression state of PDGFRB was compared with the corresponding normal tissues based on the GEPIA database including GETX normal data. ${ }^{*} \mathrm{P}<0.05$. 

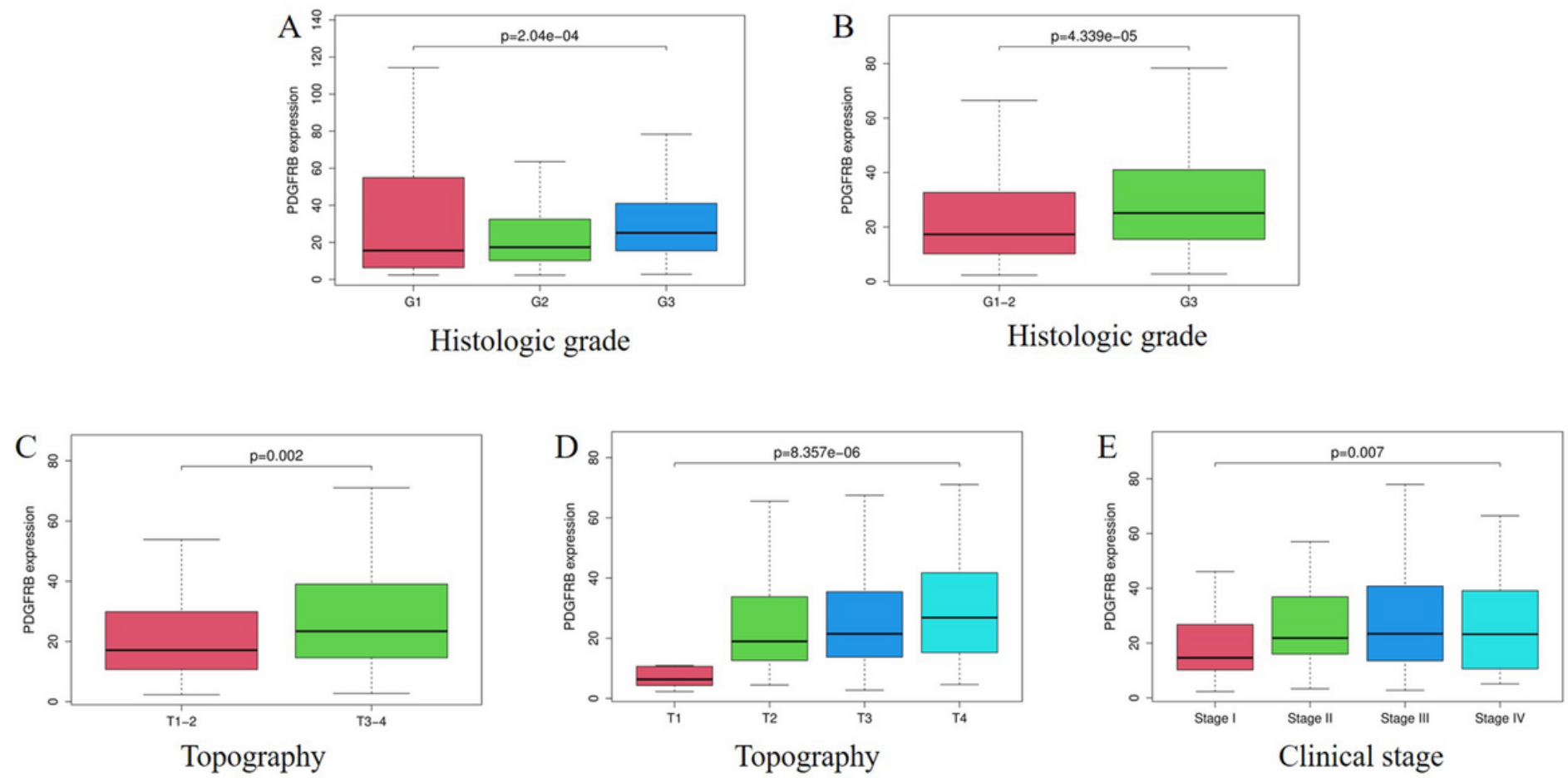

Figure 3

Correlation between PDGFRB expression and clinicopathological features. Increased PDGFRB expression was significantly correlated with histologic grade (A and B), topology (C and D) and clinical stage (E).

A

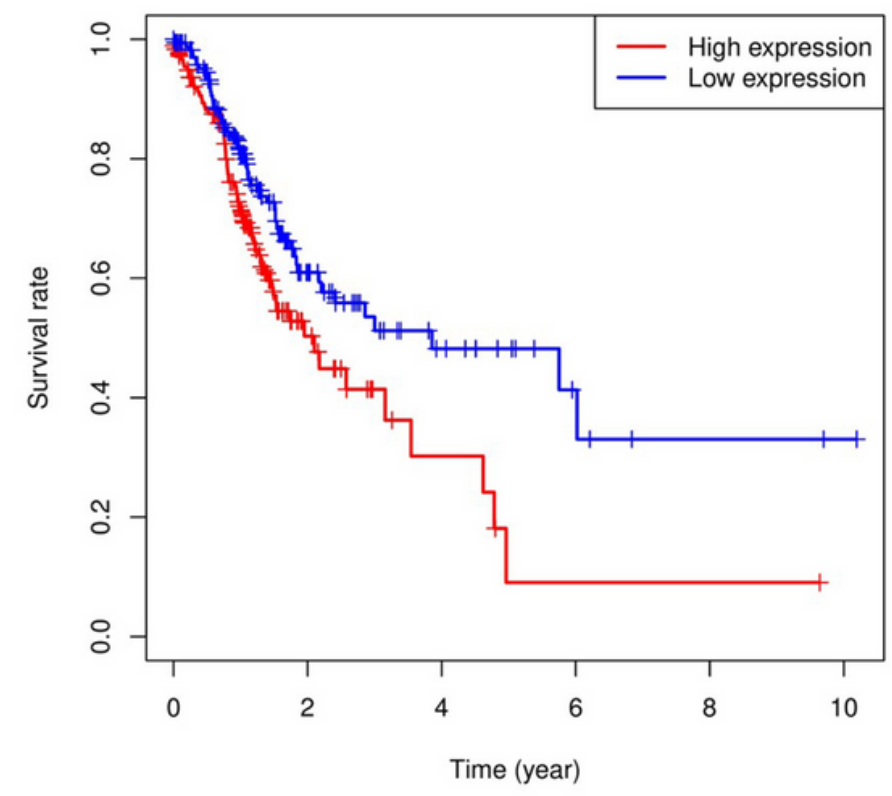

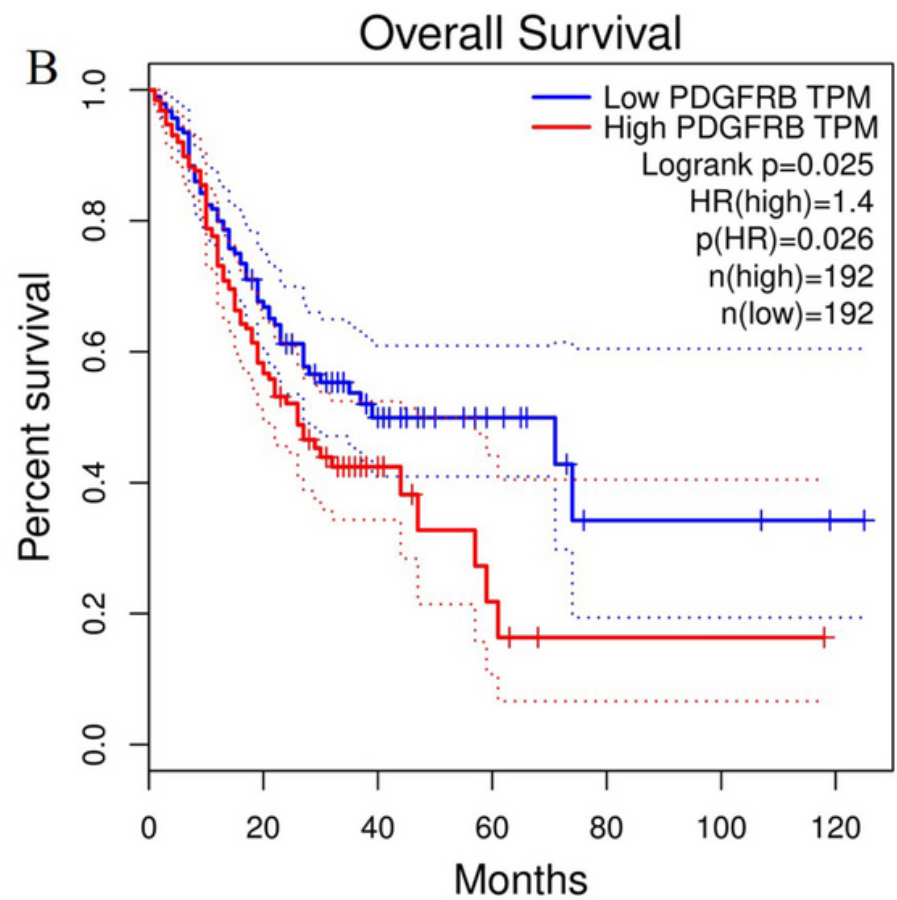

Figure 4

Survival results based on Kaplan-Meier analysis. Kaplan-Meier survival analysis showed that increased PDGFRB expression were significantly associated with worse OS. (A) the correlation between PDGFRB mRNA expression level and OS based on data from TCGA; (B) further validation of the correlation by GEPIA. 


\section{Hazard ratio}

age $\quad(N=317) \quad(1.08-2.3)$

gender

$(N=317)$

$(0.96-2.2)$

stage $3-4$ vs. $1-2$

$(N=317)$

$\left(0.77^{1.4}-2.6\right)$

$(N=317)$

$(0.68-2.0)$

T 3-4 vs. 1-2

$(0.68-2.0)$

M yes vs. no

$(N=317)$

$(1.09-4.1)$

0.019 *

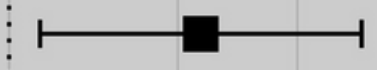

$10.019 *$

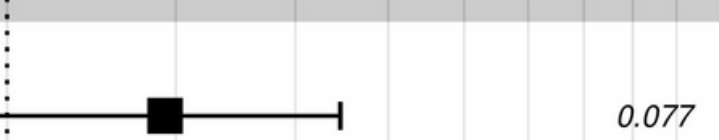

M

$\mathrm{N}$ yes vs. no

$(N=317)$

$\left(0.57^{1.1}-2.0\right)$

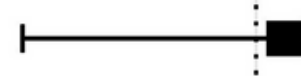

0.833

PDGFRB High vs. Low $(N=317) \quad(1.01-2.2)$

\# Events: 111; Global p-value (Log-Rank): 0.0010889

AIC: 1096.05; Concordance Index: 0.64

\section{Figure 5}

Relationship between clinicopathological features and survival results of STAD. Highly expressed PDGFRB, distant metastasis and age can independently predict unfavourable OS. 

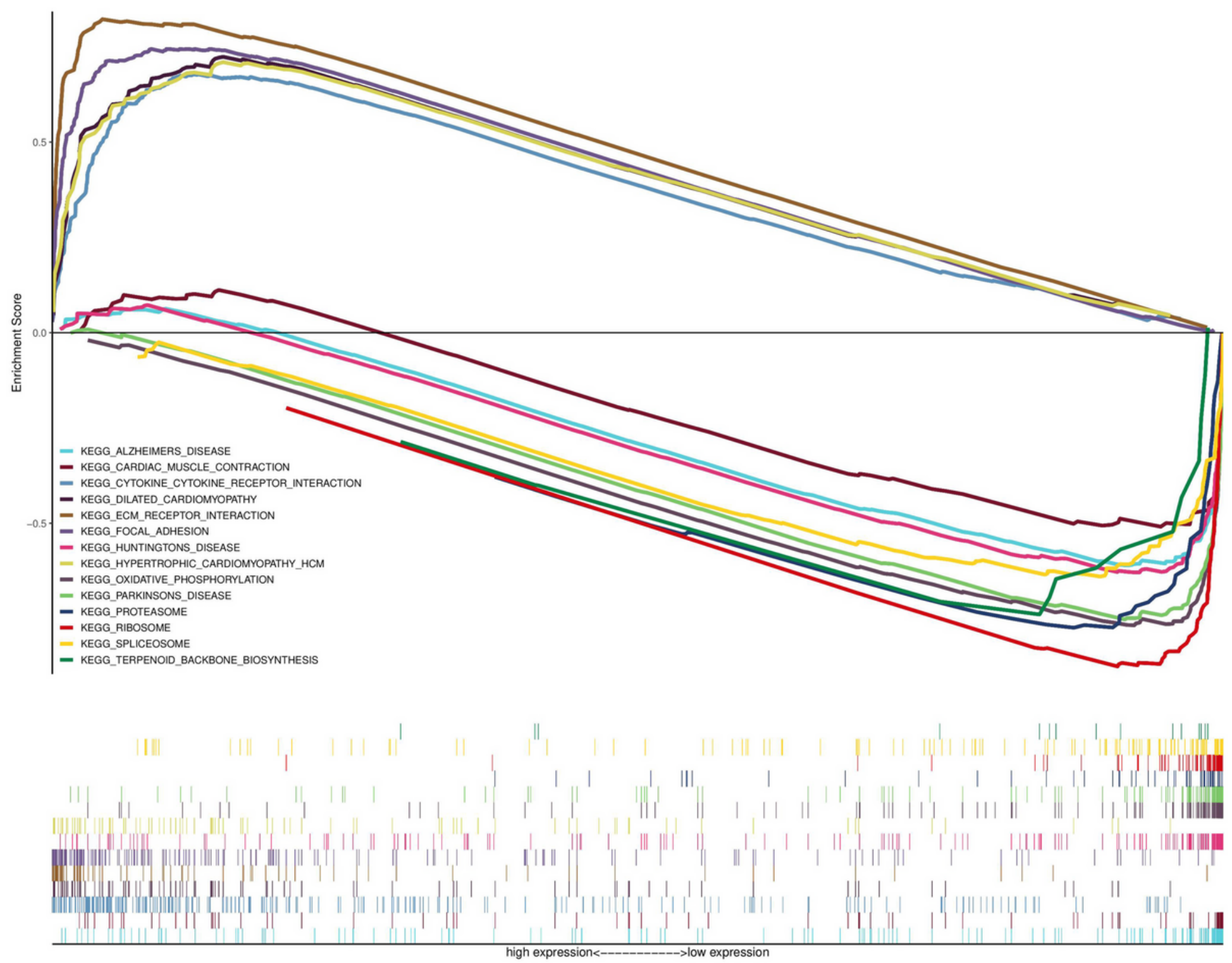

Figure 6

Enrichment plots from gene set enrichment analysis (GSEA). The GSEA results showed that the CYTOKINE-CYTOKINERECEPTOR-INTERACTION, FOCAL-ADHESION, DILATED-CARDIOMYOPATHY, ECM-RECEPTOR-INTERACTION, and HYPERTROPHIC-CARDIOMYOPATHY-HCM signalling pathways were significantly enriched in the high PDGFRB expression phenotype and PARKINSONS-DISEASE, OXIDATIVE-PHOSPHORYLATION, ALZHEIMERS-DISEASE, PROTEASOME, HUNTINGTONS-DISEASE, RIBOSOME, TERPENOID-BACKBONE-BIOSYNTHESIS, CARDIAC-MUSCLE-CONTRACTION and SPLICEOSOME signaling pathways were significantly enriched in the low PDGFRB expression phenotype. 


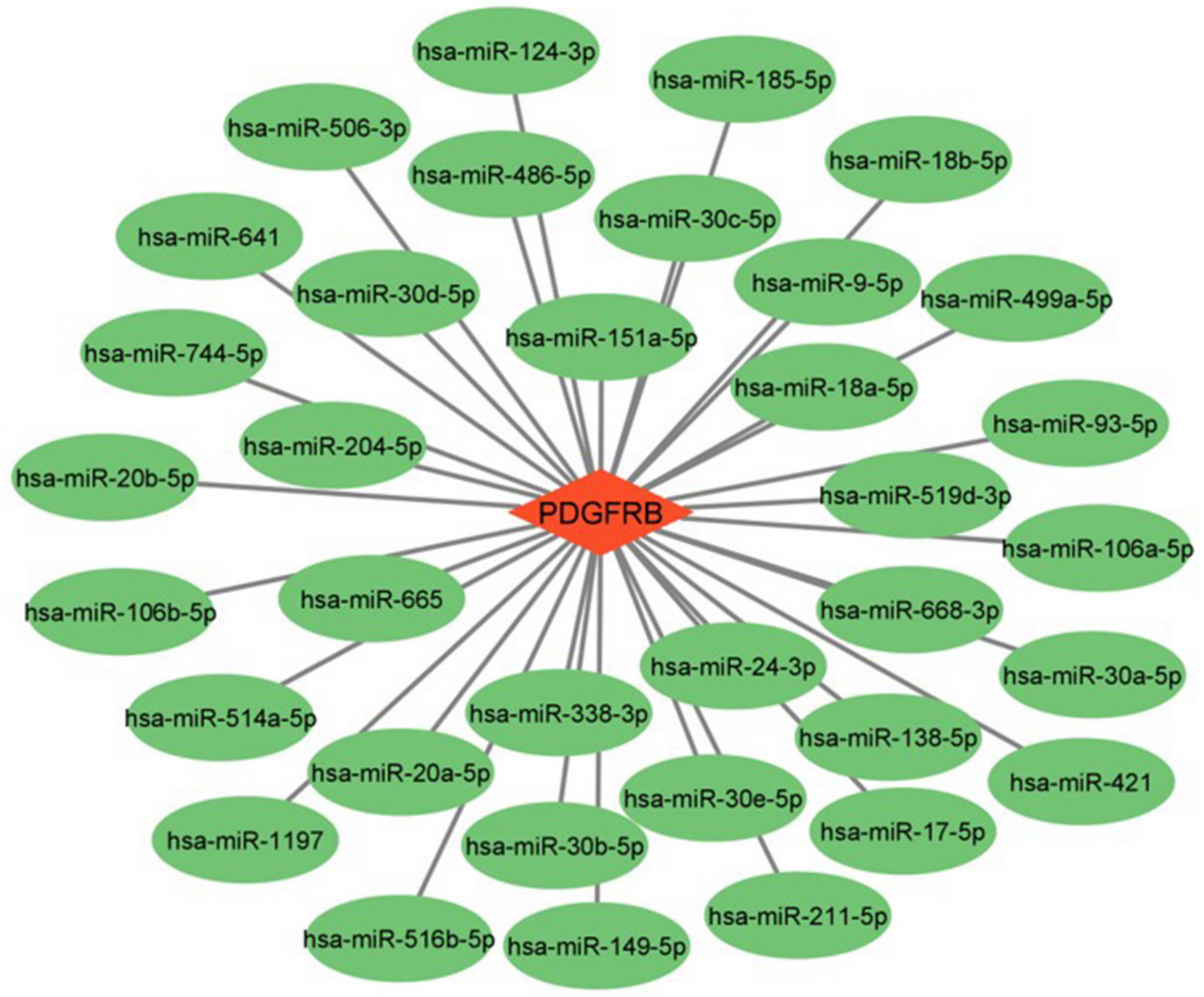

Figure 7

miRNAs-mRNA interaction network in gastric cancer. The diamond represents mRNA in red and ellipses represent miRNAs in green.
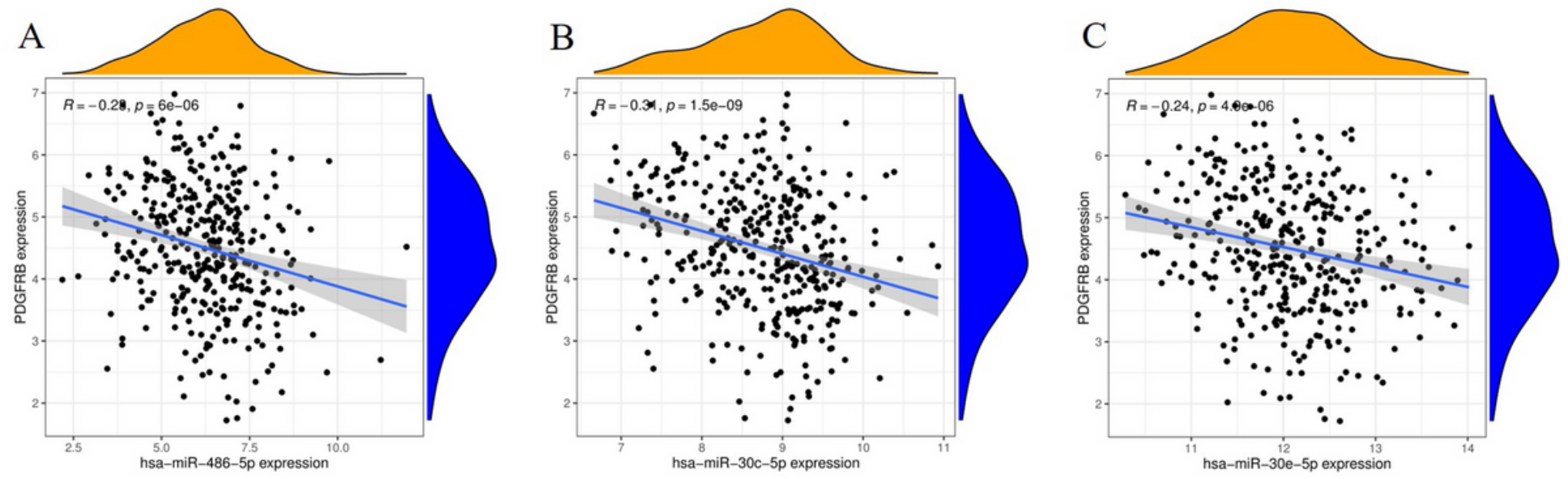
Figure 8

Relationship between PDGFRB expression and miRNAs. (A-C) hsa-miR-486-5p, hsa-miR-30c-5p and hsa-miR-30e-5p were suggested to have a significant correlation with $P D G F R B$ expression.
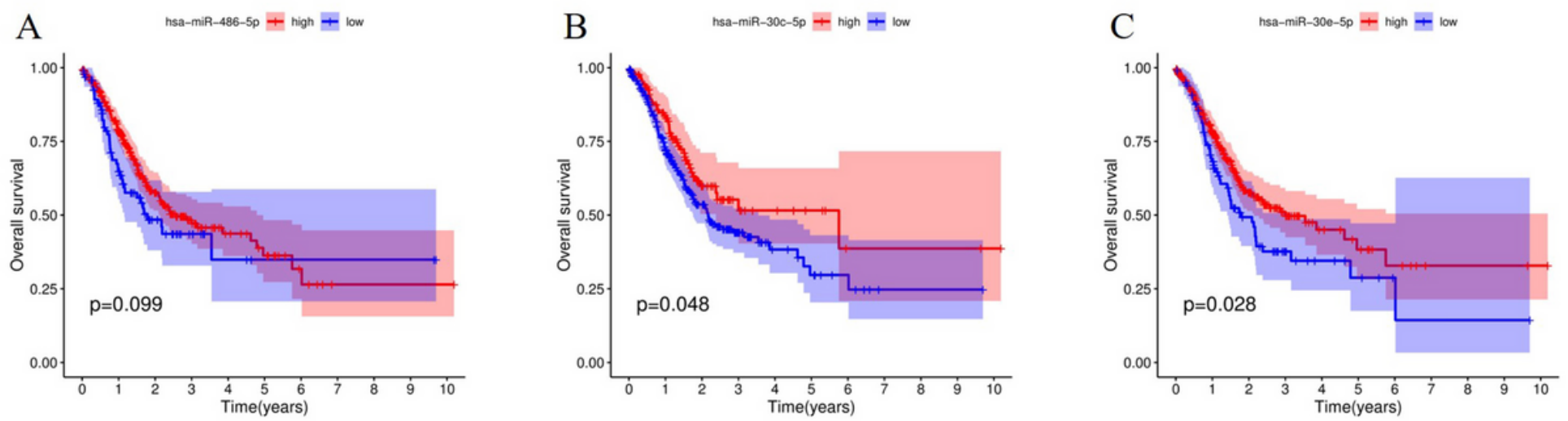

Figure 9

Survival results based on Kaplan-Meier analysis. (A) there was no a significant difference being observed between hsa-miR486-5p and OS. (B-C) Kaplan-Meier survival analysis showed that increased hsa-miR-30c-5p and hsa-miR-30e-5p expression were significantly associated with better OS, respectively. 

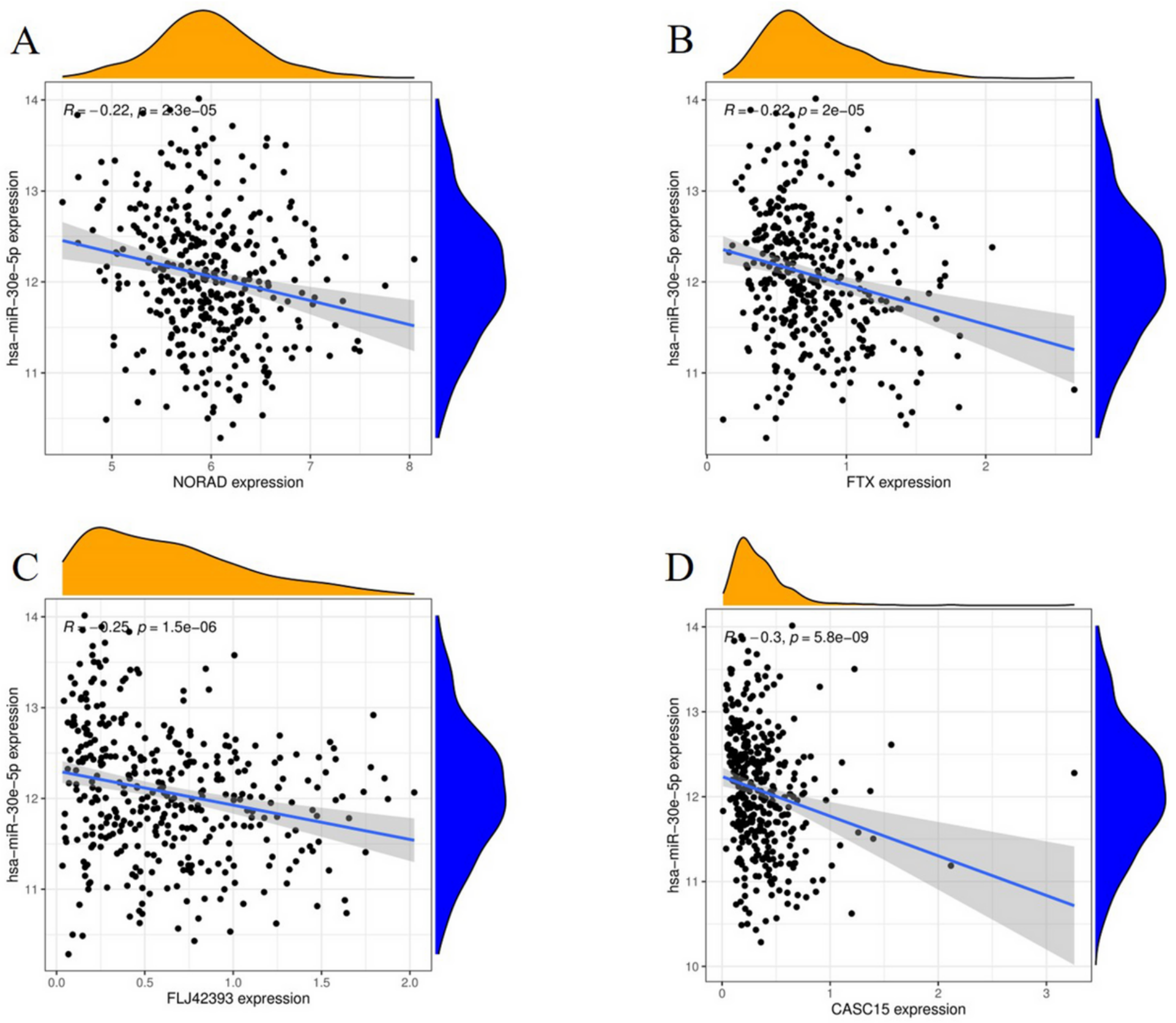

Figure 10

Relationship between hsa-miR-30e-5p and IncRNAs. (A-D) IncRNAs NORAD, FTX, FLJ42393 and CASC15 were suggested to have a significant correlation with hsa-miR-30e-5p expression. 

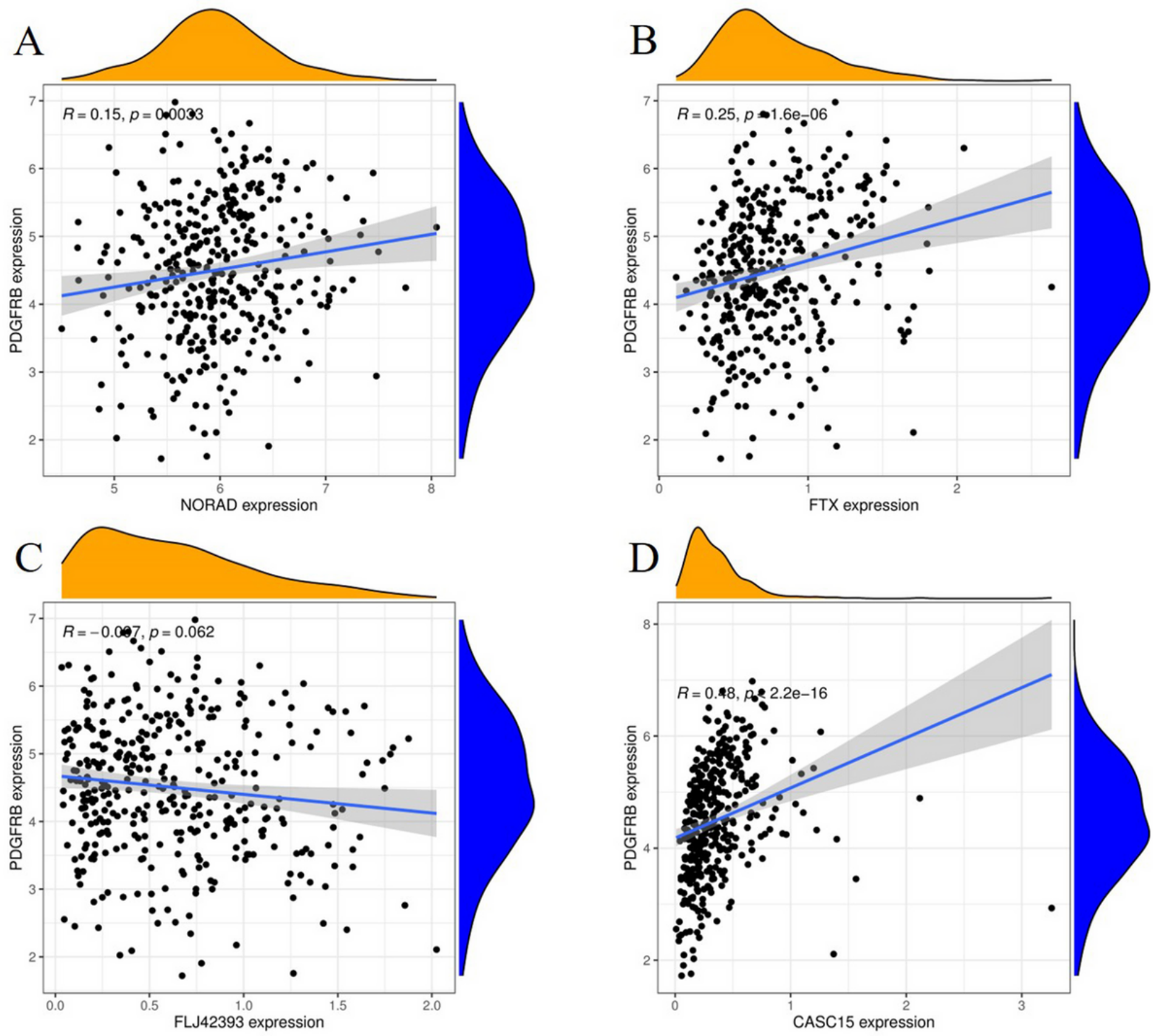

Figure 11

Relationship between PDGFRB expression and IncRNAs. (A, B and D) IncRNAs NORAD, FTX and CASC15 were suggested to have a significant correlation with PDGFRB expression. (C) there is no significant relationship between IncRNA FLJ42393 and PDGFRB expression. 
A Type 审 Normal 审 Tumor

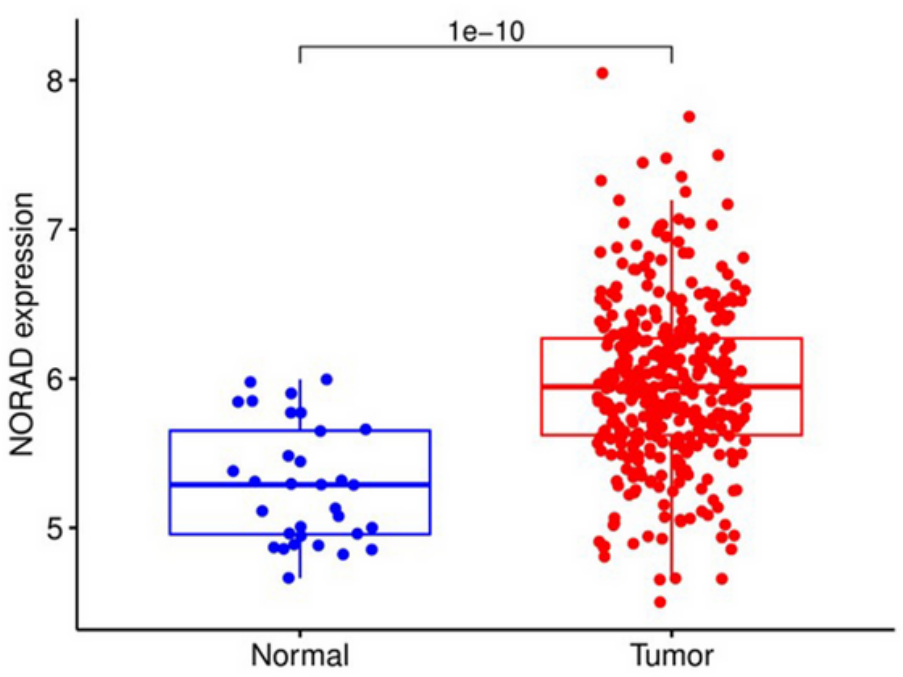

C Type 审 Normal 审 Tumor

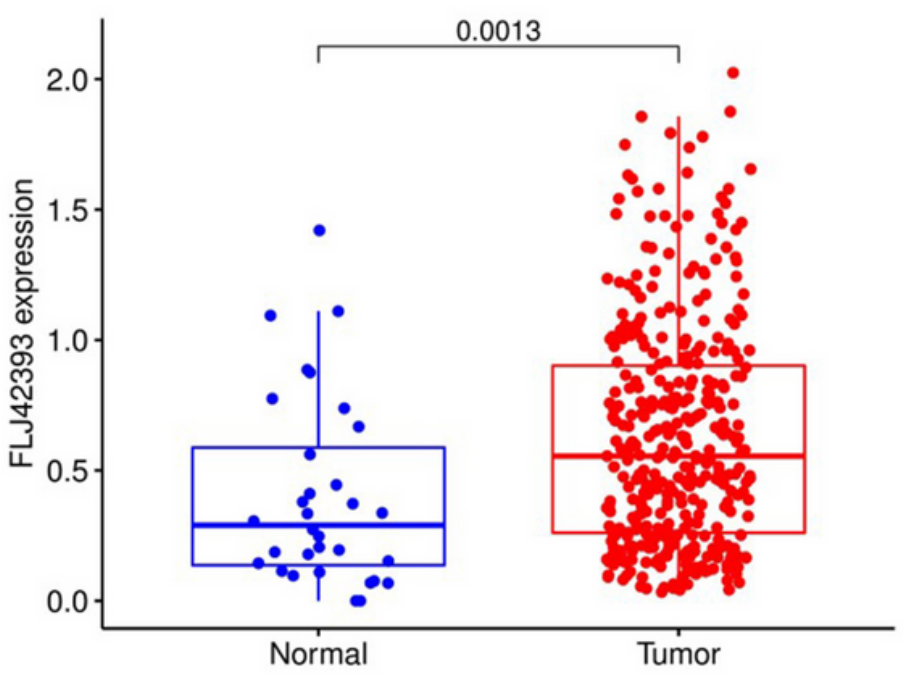

B Type 审 Normal 审 Tumor

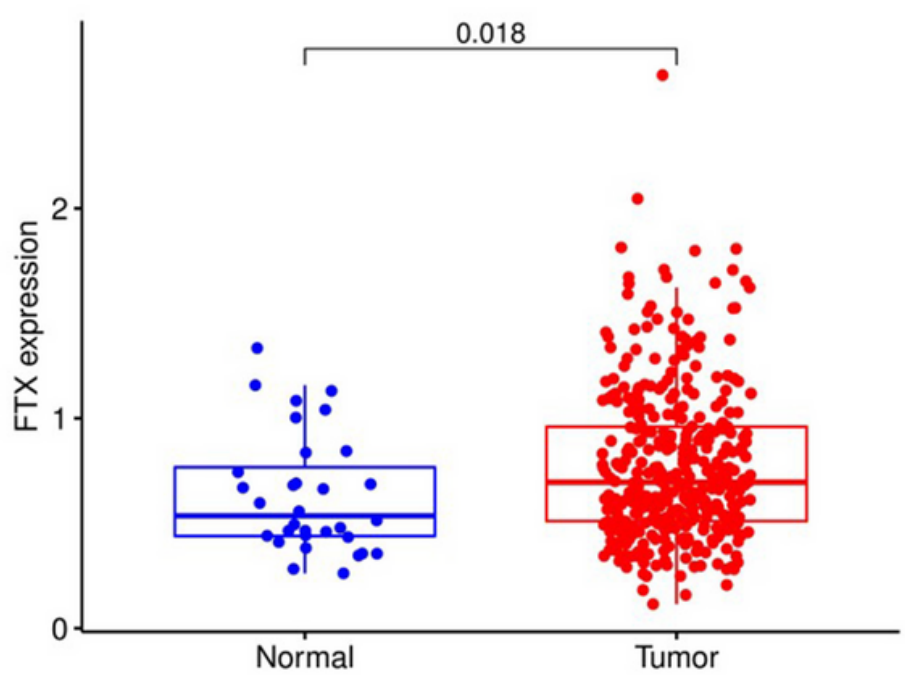

D Type 审 Normal 审 Tumor

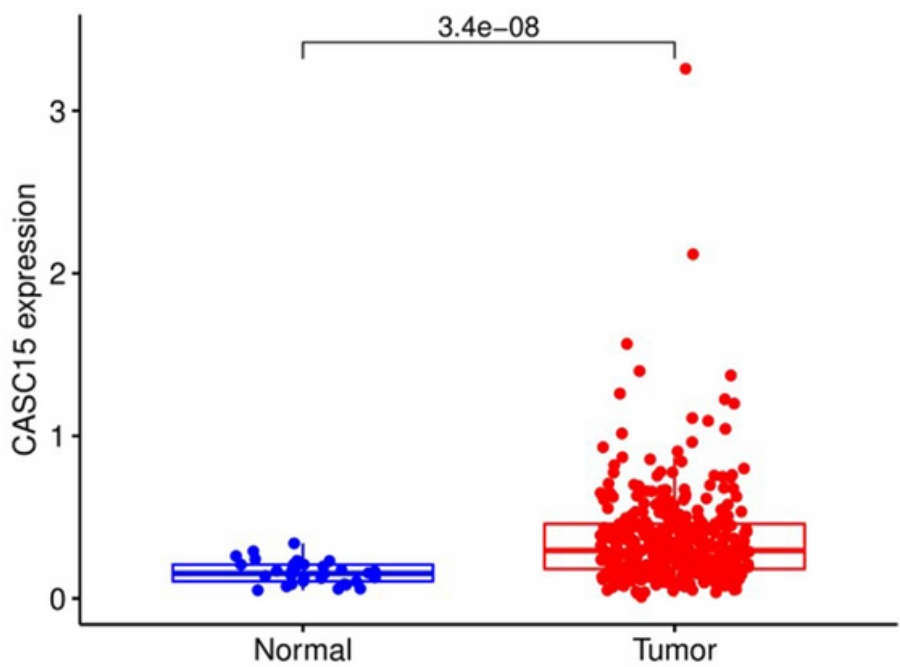

Figure 12

The expression of the four IncRNAs was increased in STAD. (A-D) IncRNAs NORAD, FTX, FLJ42393 and CASC15 all showed significantly higher expression in STAD samples than in normal samples through the Wilcoxon rank sum test. 
A

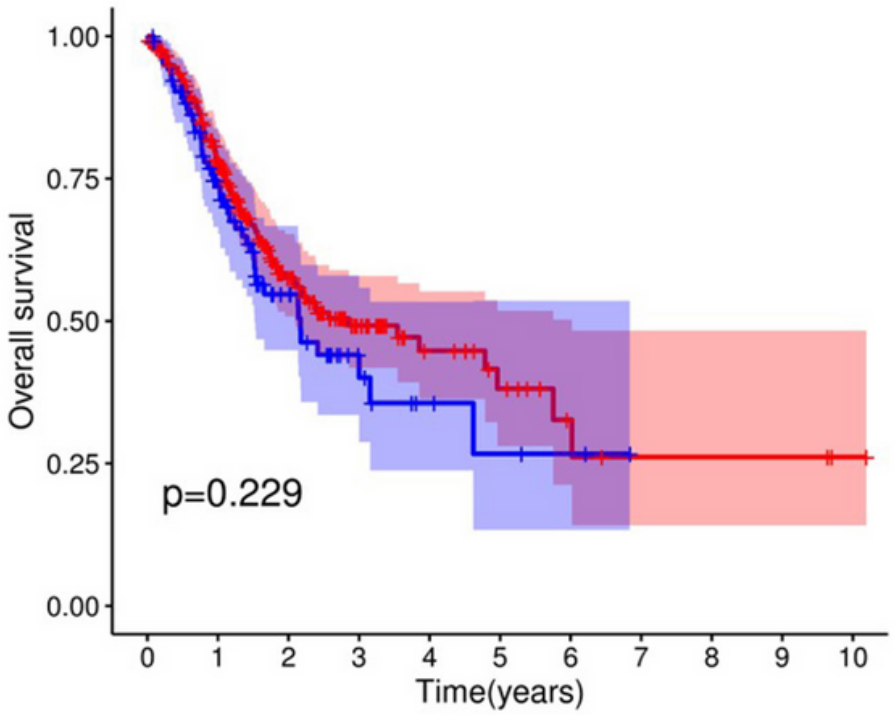

$\mathrm{C}$

$$
\text { FLJ42393 + high + low }
$$

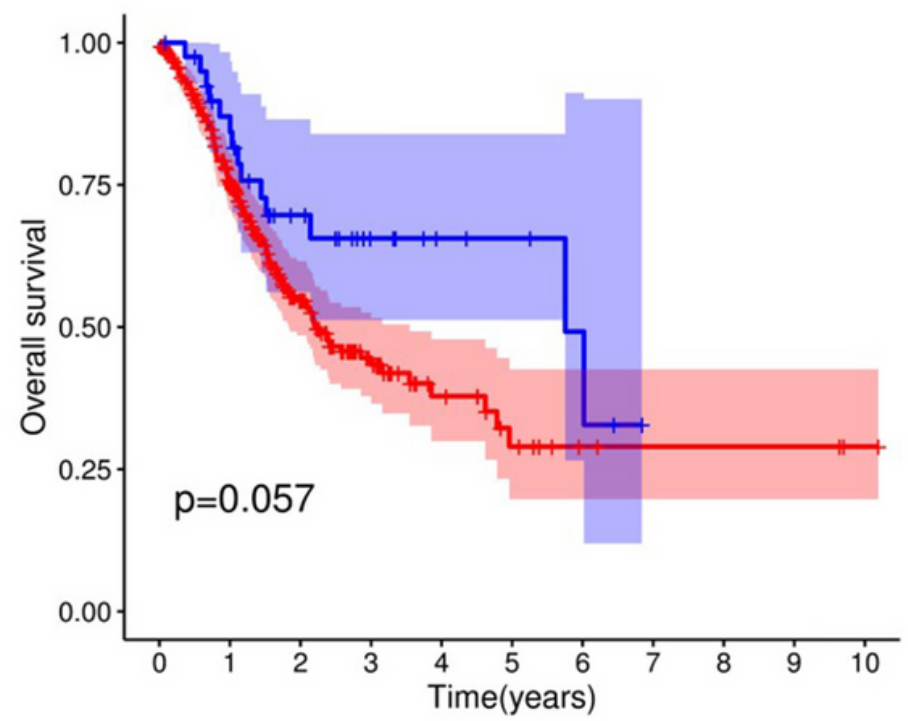

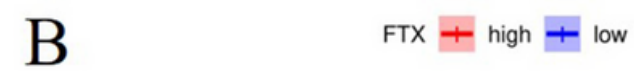

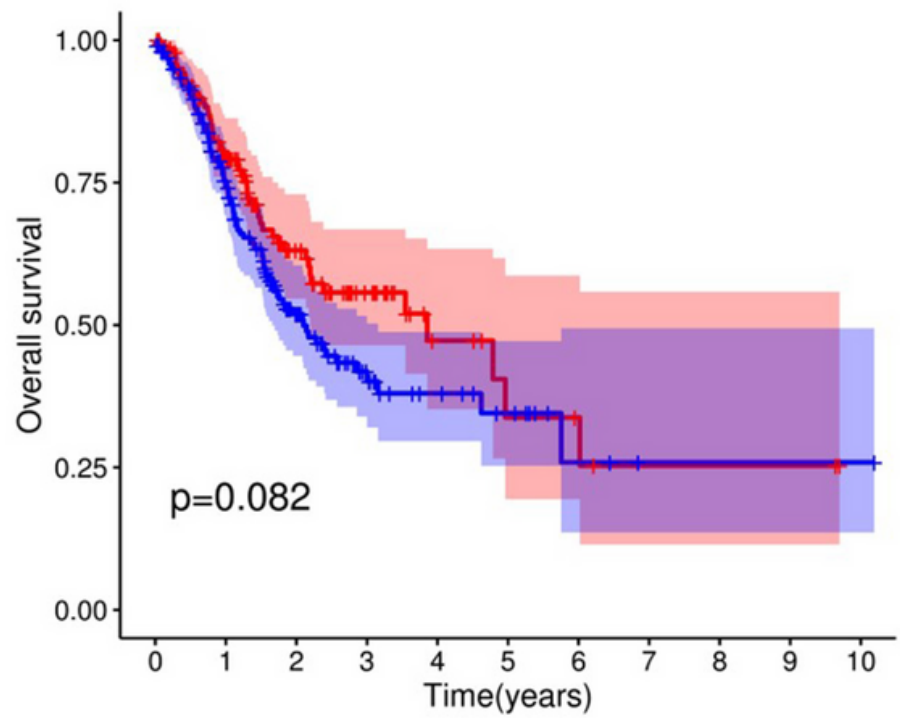

$\mathrm{D}$
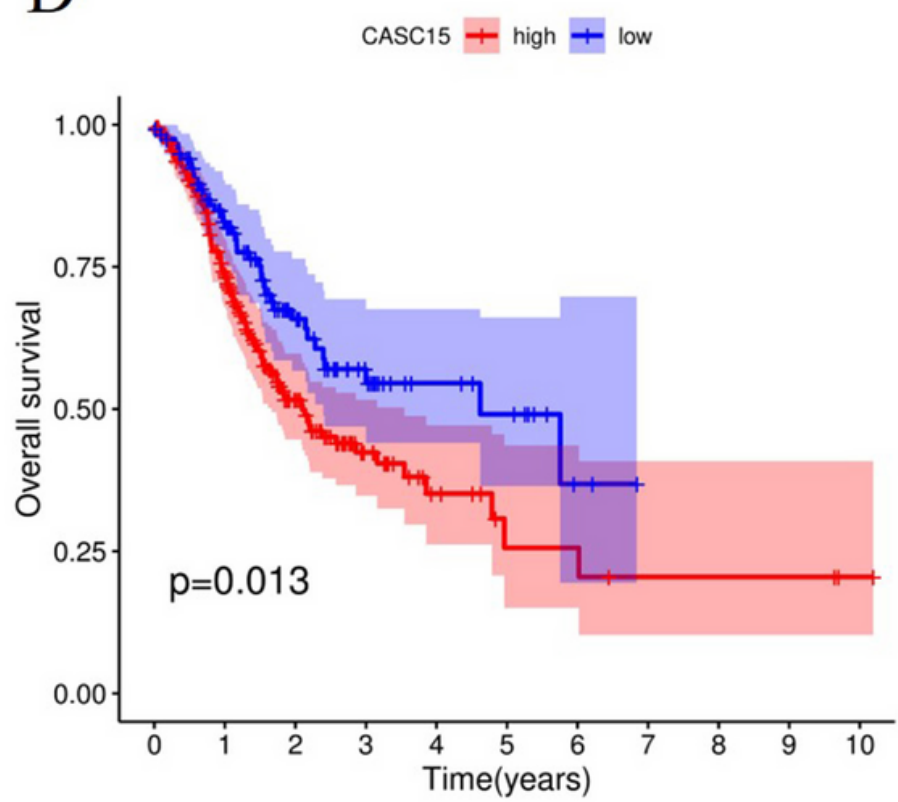

Figure 13

Kaplan-Meier survival curves for the four IncRNAs. (A) IncRNA NORAD ( $P=0.229)$; (B) IncRNA FTX $(P=0.082)$; (C) IncRNA FLJ42393 ( $P=0.057)$; (D) IncRNA CASC15 $(P=0.013)$. 


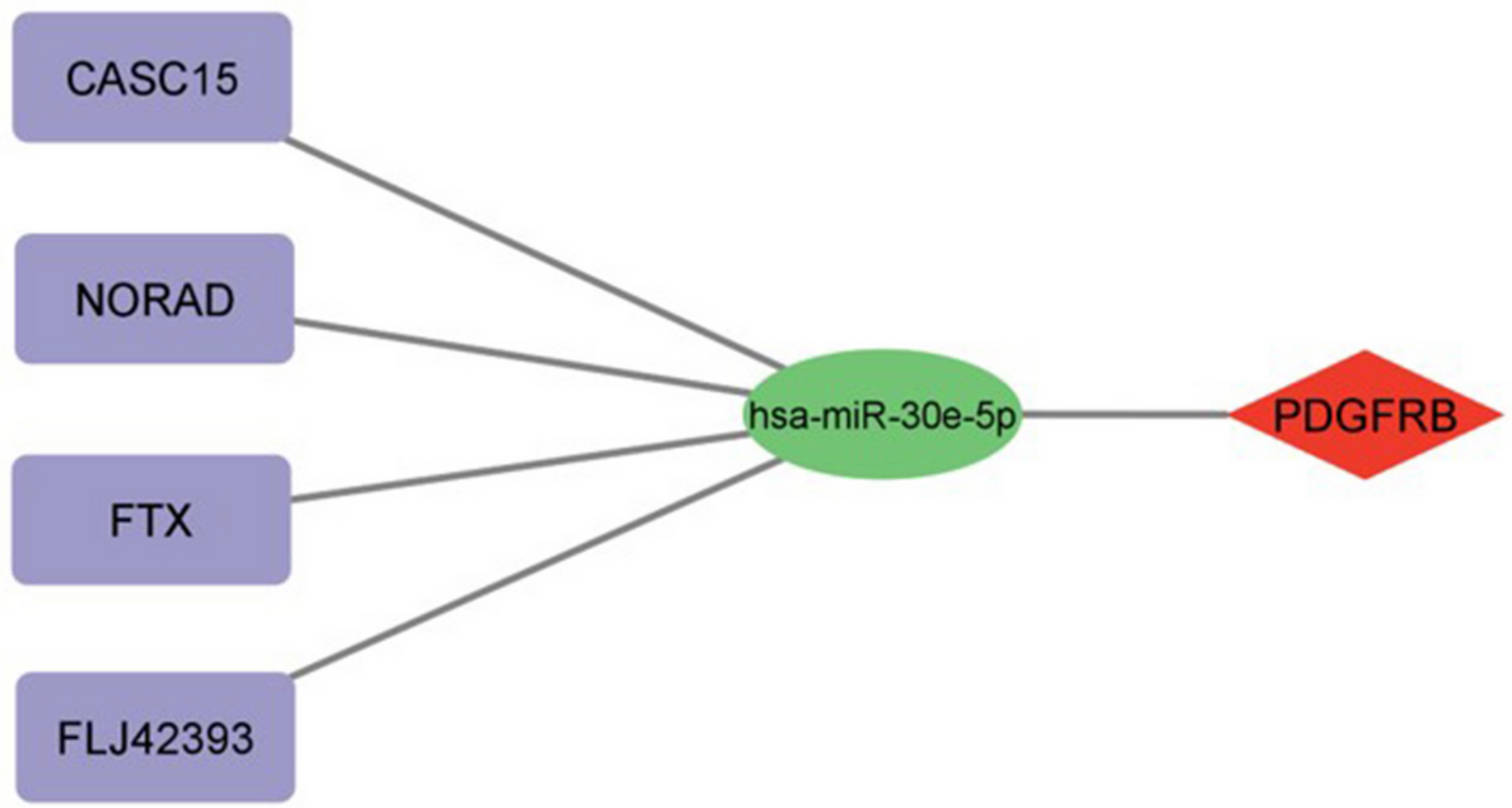

Figure 14

The IncRNAs-miRNA-target gene axis.
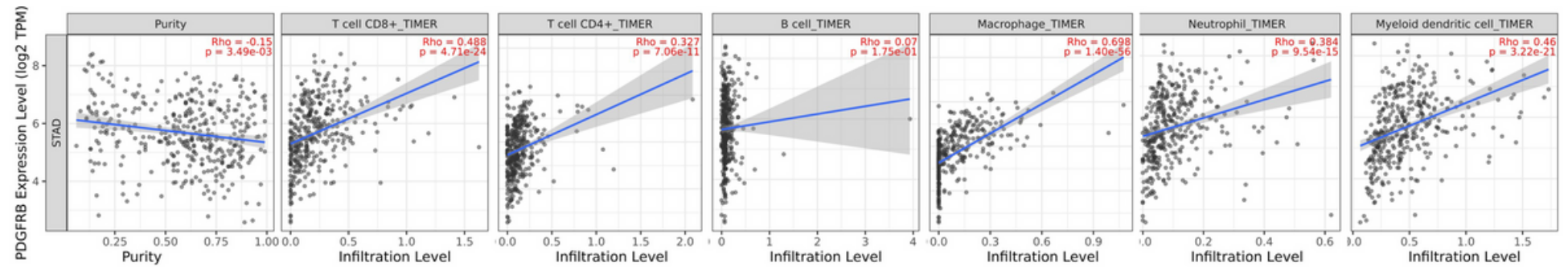

Figure 15

Correlations between PDGFRB expression and immune infiltration levels. According to the TIMER database, 5 immune cells (CD8+ T cells, CD4+ T cells, macrophages, neutrophils and dendritic cells) had significant correlations after the adjustment of purity. 

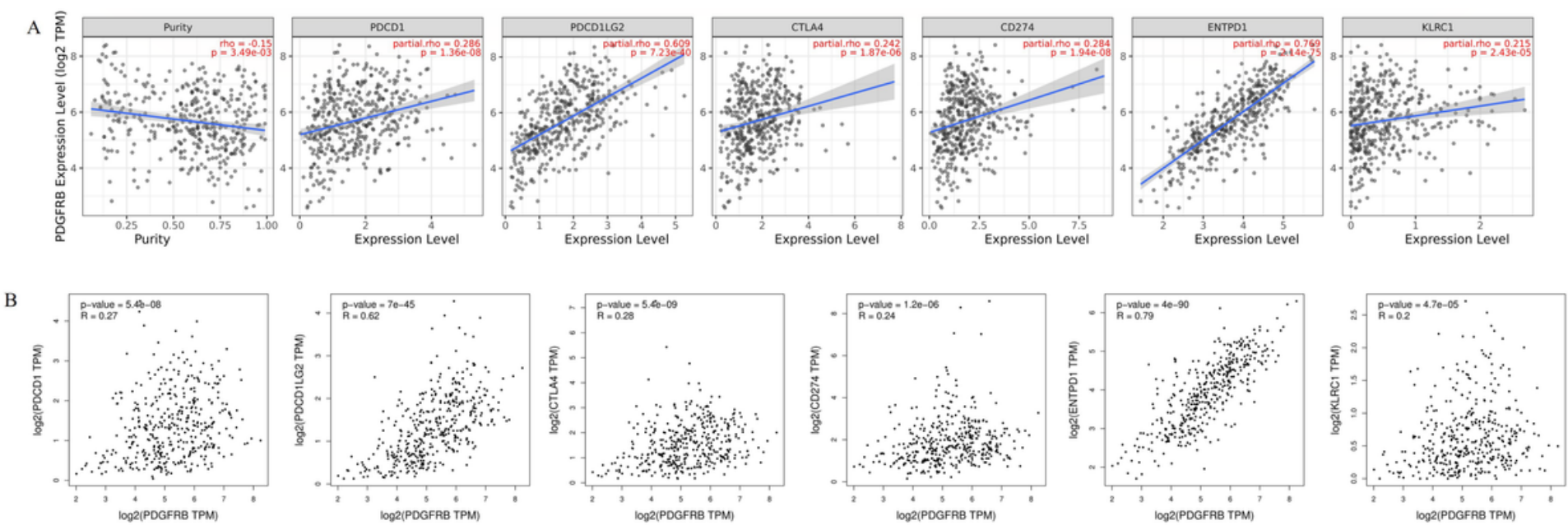

Figure 16

Correlations between six biomarkers and PDGFRB. (A) the correlations were verified through TIMER 2.0, which demonstrated that PDGFRB was significantly correlated with these immunotherapy-associated genes. (B) according to the results from GEPIA database, PDGFRB also had a significant relationship with these genes.
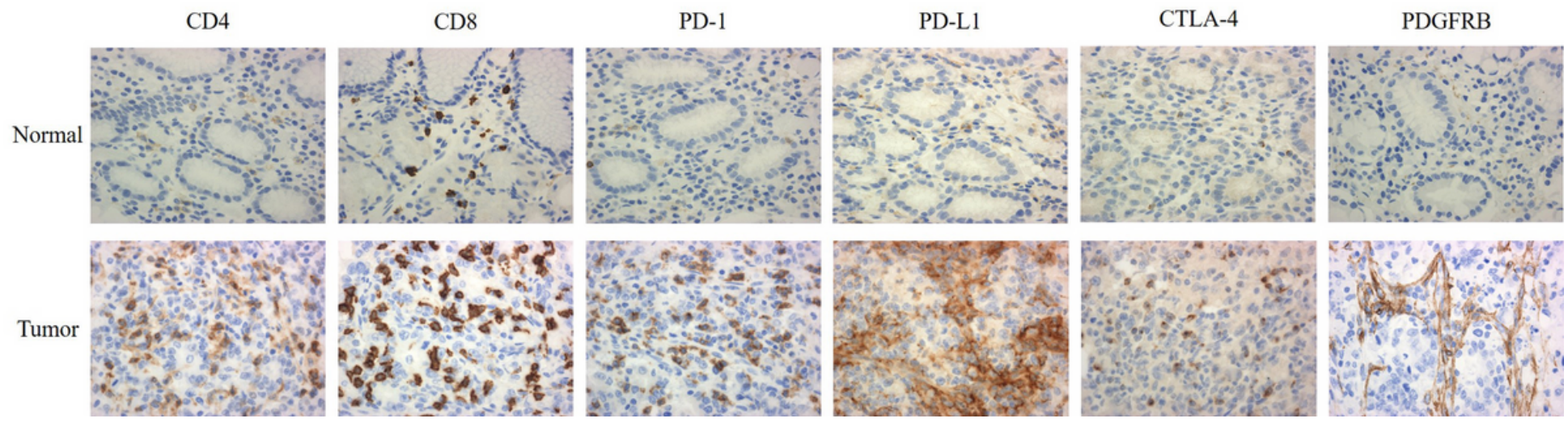

Figure 17

Immunohistochemical staining of CD4, CD8, PD-1, PD-L1, CTLA-4 and PDGFRB. In carcinoma tissue, the expression level of all proteins was higher than in normal control.

$\mathrm{P}=0.0218$

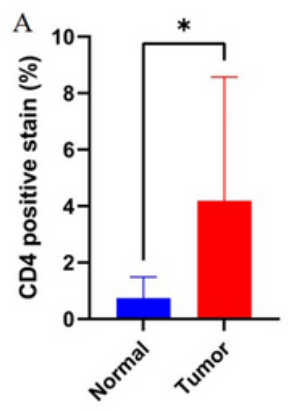

$P=0.0315$

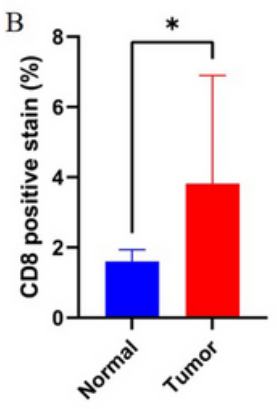

$P=0.0742$

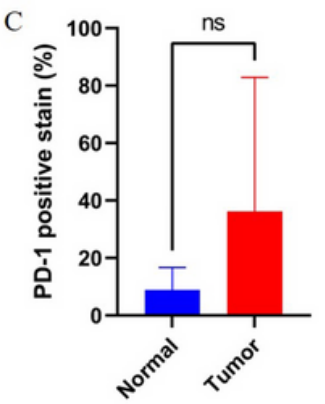

$\mathrm{P}=0.0104$

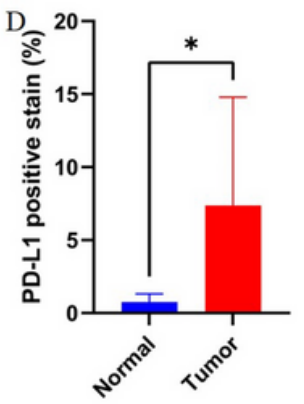

$\mathrm{P}=0.0337$

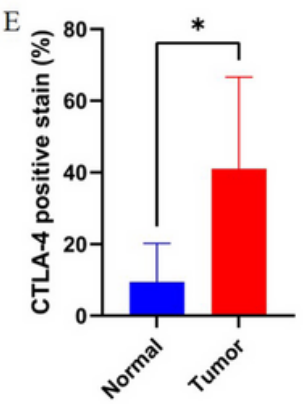

$\mathrm{P}=0.0002$

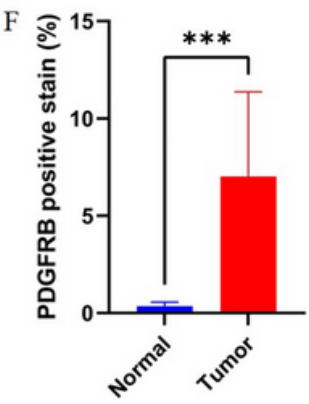

Figure 18 
The percentage of positive cells or positive regions. Expression difference of all proteins between normal and tumor tissue all reached statistical significance, except for PD-1.

A

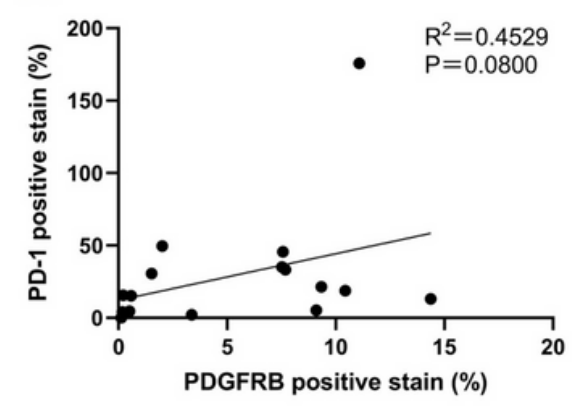

B

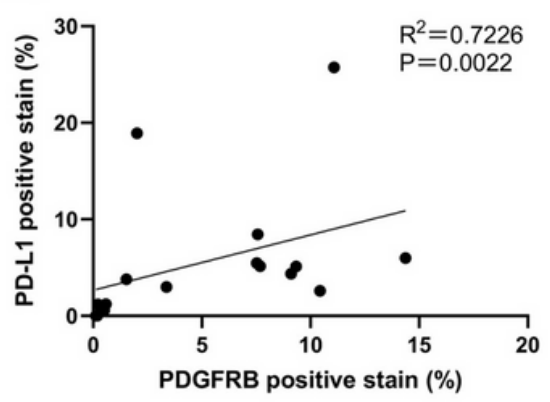

$\mathrm{C}$

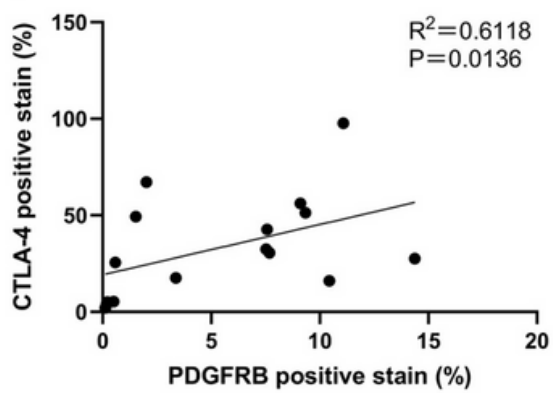

Figure 19

Correlation analysis. PDGFRB positive expression had significant correlation with the expression of PD-L1 and CTLA-4 (B and C), but no positive correlation was observed between PDGFRB and PD-1 (A).

\section{Supplementary Files}

This is a list of supplementary files associated with this preprint. Click to download.

- Supplementarytable1.docx

- Supplementarytable2.docx 